ARTICLE

\title{
Co-option of Plasmodium falciparum PP1 for egress from host erythrocytes
}

Aditya S. Paul (10 1, Alexandra Miliu (10 2, Joao A. Paulo (D) 3, Jonathan M. Goldberg' , Arianna M. Bonilla1, Laurence Berry², Marie Seveno², Catherine Braun-Breton², Aziz L. Kosber (1) 1, Brendan Elsworth (1) Jose S. N. Arriola (1) ', Maryse Lebrun², Steven P. Gygi ${ }^{3}$, Mauld H. Lamarque (i) ${ }^{2,4 凶}$ \& Manoj T. Duraisingh (1) ${ }^{1,4 凶}$

Asexual proliferation of the Plasmodium parasites that cause malaria follows a developmental program that alternates non-canonical intraerythrocytic replication with dissemination to new host cells. We carried out a functional analysis of the Plasmodium falciparum homolog of Protein Phosphatase 1 (PfPP1), a universally conserved cell cycle factor in eukaryotes, to investigate regulation of parasite proliferation. PfPP1 is indeed required for efficient replication, but is absolutely essential for egress of parasites from host red blood cells. By phosphoproteomic and chemical-genetic analysis, we isolate two functional targets of PfPP1 for egress: a HECT E3 protein-ubiquitin ligase; and $\mathrm{GC} \alpha$, a fusion protein composed of a guanylyl cyclase and a phospholipid transporter domain. We hypothesize that PfPP1 regulates lipid sensing by $\mathrm{GC} \alpha$ and find that phosphatidylcholine stimulates PfPP1-dependent egress. PfPP1 acts as a key regulator that integrates multiple cell-intrinsic pathways with external signals to direct parasite egress from host cells.

\footnotetext{
${ }^{1}$ Department of Immunology and Infectious Diseases, Harvard T. H. Chan School of Public Health, Boston 02115 MA, USA. ${ }^{2}$ Laboratory of Pathogen Host Interaction (LPHI), UMR5235, Centre National de la Recherche Scientifique (CNRS), Université de Montpellier, 34095 Montpellier, France. ${ }^{3}$ Department of Cell Biology, Harvard Medical School, Boston 02115 MA, USA. ${ }^{4}$ These authors contributed equally: Mauld H. Lamarque, Manoj T. Duraisingh.

凶email: mauld.lamarque@umontpellier.fr; mduraisi@hsph.harvard.edu
} 
$\mathrm{M}$ alaria parasites from the genus Plasmodium follow an unusual developmental program during infection of erythrocyte host cells, utilizing a non-canonical style of asexual proliferation known as schizogony to undergo multiple cycles of nuclear replication before a single cytokinesis event to form merozoites ${ }^{1,2}$. Merozoites infect new host cells to initiate new intraerythrocytic developmental cycles (IDC) and sustain proliferation, achieved only by egress from host cells for release into circulation. Protein phosphorylation in parasites is developmentally regulated in blood-stage growth ${ }^{3}$, and genetic studies show that roughly one-half of the Plasmodium protein kinase and protein phosphatase genes are essential for the $\mathrm{IDC}^{4-8}$.

Protein Phosphatase 1 (PP1) is a highly conserved and ubiquitous enzyme in eukaryotes that regulates mitotic exit and cytokinesis $^{9-11}$. With functions also in non-cell cycle-related processes (reviewed in ${ }^{12}$ ), PP1 is a dominant contributor to total cellular phosphatase activity ${ }^{13}$. For the Plasmodium falciparum homolog of PP1 (PfPP1) $)^{14}$, genetic evidence for likely essentiality ${ }^{5,7}$, high levels of expression ${ }^{15,16}$ (Supplementary Fig. 1a), and the identification of several binding proteins (e.g. Refs. ${ }^{17-21}$ ), suggest multiple functions in asexual, blood-stage parasites.

We used a reverse genetic approach to carry out a functional analysis of PfPP1 in asexual proliferation of P. falciparum. With conditional expression of the endogenously expressed enzyme, we establish essential functions for PfPP1 during intraerythrocytic development and at egress. Phosphoproteomic and chemicalgenetic analyses of essential PfPP1 activity at egress indicate regulation of the functions of a HECT-family E3 ligase, and a putative phospholipid transporter fused to a guanylyl cyclase utilized for host cell rupture. Based on chemical-genetic and biochemical evidence, we propose that PfPP1 regulates translocation of phosphatidylcholine across the phospholipid transporter and stimulates cGMP synthesis for egress.

\section{Results}

PfPP1 in parasite development and host cell egress. To investigate PfPP1-mediated regulation of the IDC and parasite proliferation, we initiated a reverse genetic analysis. With a transgenic $P$. falciparum line expressing a triple-hemagglutinin $\left(\mathrm{HA}_{3}\right)$ tag at the $3^{\prime}$-end of the endogenous $p f p p 1$ gene (Supplementary Fig. 1b, c), we found that PfPP1 protein is expressed throughout development, with evidence for upregulation in the latter half of the 48-h IDC (Fig. 1a; Supplementary Fig. 1d). In agreement with cell biological analysis in the rodent malaria parasite Plasmodium berghe $i^{19}$ and subcellular fractionation in $P$. falciparum $^{21}$, we observed by immunofluorescence analysis PfPP1 near the nucleus and in the parasite cytoplasm throughout the IDC (Supplementary Fig. 1e). Cell cycle stage-dependent compartmentalization of PP1 has been reported in lower eukaryotes, consistent with distinct functions for the enzyme ${ }^{22}$. To assess the function of PfPP1 in blood-stage proliferation, we generated a transgenic line of $P$. falciparum for inducible knockout of the $p f p p 1$ gene $(p f p p 1-i K O)$, based on the dimerizable Cre recombinase (DiCre) system (Supplementary Fig. 1b, f-h) ${ }^{23,24}$. Induction of $p f p p 1$-knockout by rapamycin treatment early in the IDC, $\sim 3-5 \mathrm{~h}$ post-invasion (hpi), results in strong reduction of PfPP1 protein levels by $\sim 30$ hpi (Fig. 1b; Supplementary Fig. 1b, h). By following DNA replication through the IDC, we found that earlyiKO of $p f p p 1$ delays parasite development before resulting in the accumulation of multinucleate schizont forms, blocked prior to egress (Fig. 1c, Supplementary Figs. 1i and 2a). Reverse genetic analysis by inducible knockout thus establishes the essentiality of PfPP1 for asexual proliferation.
To investigate PfPP1 function at specific times through the IDC, we used a transgenic P. falciparum line for conditional knockdown (Supplementary Fig. 3a-c). Knockdown of PfPP1 fused to a Destabilization Domain (DD)-tag, induced through depletion in culture of the DD-stabilizing small molecule Shield-1 (Shld1) ${ }^{25-27}$, confirms essentiality of the enzyme to blood-stage parasites (Fig. 1d, e; Supplementary Figs. 2b and 3d, e). In this transgenic line, we also observed PfPP1 protein throughout the IDC, with expression more pronounced at later stages (Supplementary Fig. 3f). To map the time of function of PfPP1, we induced knockdown at different stages of the IDC and measured ensuing DNA replication. We measured phenotypes with knockdown induced at the immature ring stage preceding the growth phase (4 hpi), at trophozoites before the onset of DNA replication (22 hpi), and early in schizogony (33 hpi) (Fig. 1f). With destabilization of PfPP1-DD in rings, we observed substantial defects in DNA replication (Fig. 1f; Supplementary Figs. $2 \mathrm{c}$ and $3 \mathrm{~g}$ ). Knockdown induced in trophozoites permits DNA replication (Fig. 1f; Supplementary Figs. 2c and $3 \mathrm{~h}$ ) but results in parasites with reduced numbers of nuclear centers (Fig. 1g; Supplementary Fig. 3i), suggesting defects in nuclear division. Electron microscopy of PfPP1-DD knockdown parasites indicates failure to complete the terminal mitosis and cytokinesis step of the IDC (Supplementary Fig. 3j), consistent with cell cycle-regulatory functions for PfPP1 conserved with non-parasitic eukaryotes ${ }^{10,11,28}$. Defects upstream of cytokinesis with PfPP1DD knockdown are supported by immunofluorescence analysis: we observe that the inner membrane complex (IMC, antigen $P f G A P 45)$ that separates replicated, intracellular parasites fails to form (Supplementary Fig. 3k).

To map functions for PfPP1 late in the IDC, we induced knockdown in schizonts ( $\sim 44 \mathrm{hpi})$, revealing an acute requirement for the phosphatase for egress after complete DNA replication and nuclear segregation (Fig. 1h, i; Supplementary Fig. 2c). Knockdown elicits a complete block in host cell egress and erythrocyte re-invasion (Fig. 1h; Supplementary Figs. 2d and 3l, m). Partial knockdown late in the IDC elicits sublethal defects in egress without additional defects observed in the further transition to invaded erythrocytes (egress-to-invasion) (Fig. 1j; Supplementary Fig. 2d), suggesting a specific function in egress.

Requirement of PfPP1 at an early step of egress. The effects of late PfPP1-DD knockdown are recapitulated in the $p f p p 1-i K O$ line with induction of Rapa-mediated iKO later in the IDC (30 hpi, Supplementary Fig. 4a), resulting in depletion of PfPP1 protein at the late schizont stage (Fig. 2a). Late iKO blocks passage to new erythrocytes without defects in DNA replication or nuclear segregation (Fig. 2b, c; Supplementary Figs. 2a and 4b). Electron microscopy shows that parasites having undergone late $p f p p 1-$ $i K O$ display gross morphology typical of maturation, including intact erythrocyte membranes, parasitophorous vacuoles that house parasites, and individual parasite cells physically distinguished by plasma membranes indicating the completion of cytokinesis (Fig. 2d). Immunofluorescence microscopy to image markers for the parasite plasma membrane (antigen PfMSP1) and the underlying IMC (antigen PfMTIP1) confirms that iKO of $p f p p 1$ does not perturb cytokinesis and segregation of these structures into replicated parasites (Fig. 2e, f; Supplementary Fig. 4c, d). Immunofluorescence shows also that secretory organelles utilized for invasion, micronemes (antigen PfAMA1), and rhoptry necks (antigen PfRON4), form normally with iKO of $p f p p 1$ (Fig. 2e, f). In the PfPP1-DD line, immunofluorescence and electron microscopy show that parasites induced for knockdown late in the IDC undergo cytokinesis (IMC antigen PfGAP45) and 
a

$$
\text { pfpp1-ha } 3
$$
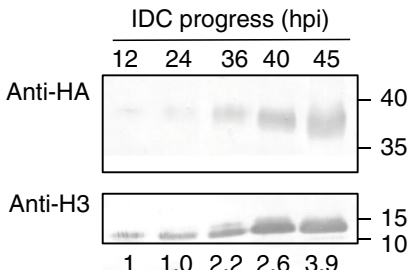

b

pfpp1-iKO

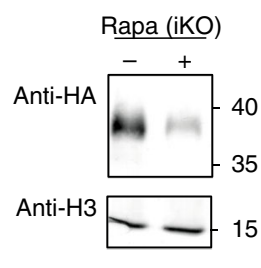

c

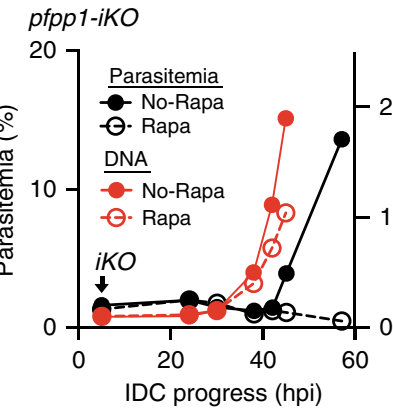

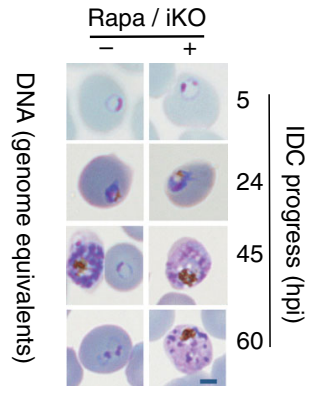

d

PfPP1-DD

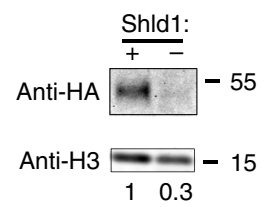

PfPP1-DD:

mid-stage induction

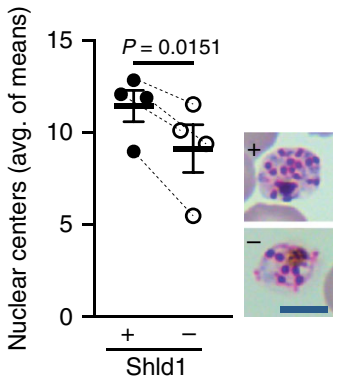

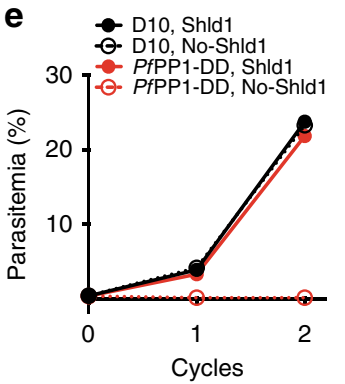

h

PfPP1-DD

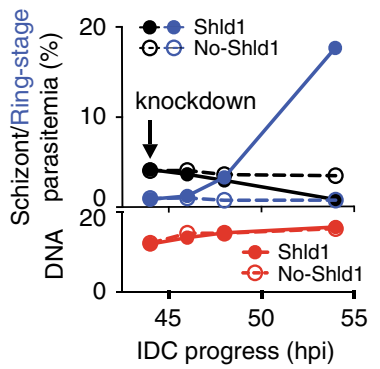

f $\quad P f \mathrm{PP} 1-\mathrm{DD}$
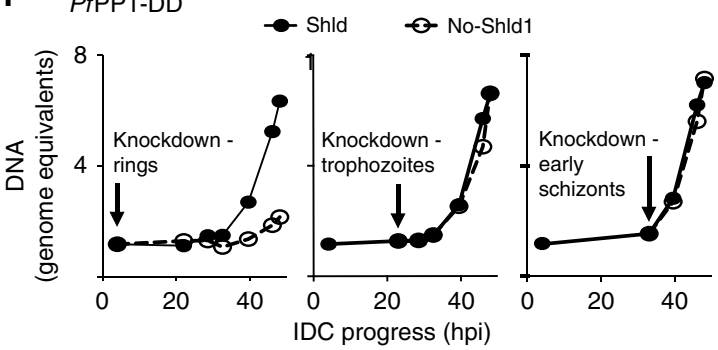

i

PfPP1-DD: late-stage induction

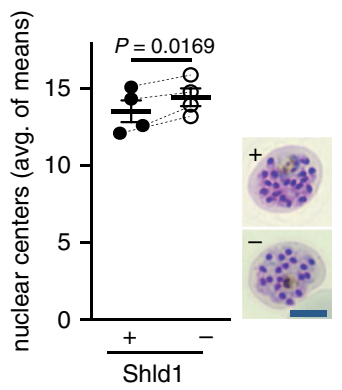

j

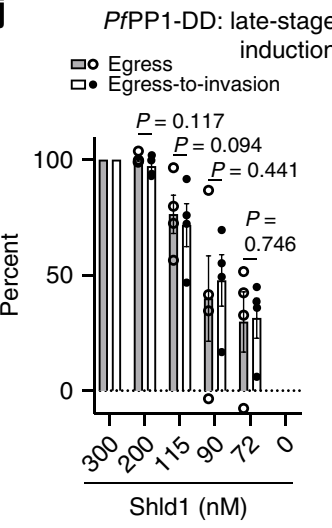

Fig. 1 Isolating an essential PfPP1 function late in the intraerythrocytic developmental cycle. a PfPP1-HA $A_{3}$ expression during intraerythrocytic development (hours post-invasion, hpi), assessed by immunoblot. Relative PfPP1 levels (below lanes) are normalized to histone H3. Representative of 3 experiments. Molecular mass in $\mathrm{kDa}$. b iKO of pfpp1 was initiated at $5 \mathrm{hpi}$ with rapamycin (Rapa) and protein levels were assessed by immunoblot at $30 \mathrm{hpi}$ (sample processing control on separate gel). Representative of 2 experiments. Molecular mass in kDa. c Left: Parasitemia and DNA synthesis over the IDC following +/-Rapa-treatment at 5 hpi in pfpp1-iKO parasites, monitored by flow-cytometry. Mean of 3 technical replicates. Representative of 4 experiments. Right: Images of parasites along the IDC, following +/-Rapa-treatment at 5-hpi. Scale bar: $2 \mu$ m. d HA-tagged PfPP1-DD protein from schizont-stage parasites ( $48 \mathrm{hpi}$ ) grown $+/-$ Shld1 for $6 \mathrm{~h}$, assessed by immunoblot. Molecular mass in kDa. Representative of 1 experiment. e Proliferation of PfPP1-DD and parental D10 parasites (wild type), +/-Shld1, monitored by flow-cytometry. Knockdown was induced in cycle-zero. Mean of 2 technical replicates. Representative of 2 experiments. $\mathbf{f}$ DNA replication in PfPP1-DD parasites following knockdown at the indicated timepoints, monitored by flow-cytometry. Mean of 2 technical replicates. Representative of 4 experiments for ring-stage induction, 2 experiments for trophozoitestage induction, and 2 experiments for early schizont-stage induction. $\mathbf{g}$ Left: Nuclear centers in terminally developed PfPP1-DD parasites, assessed by light microscopy. Knockdown induced at 22-30 hpi. Mean \pm s.e.m.; $n=4$ experiments; two-tailed $t$ test. Right: Representative images of terminal parasites +/-Shld1. Scale bar: $5 \mu \mathrm{m}$. h Top: Schizont and ring-stage parasites monitored by flow-cytometry following induction of PfPP1-DD knockdown (+/-Shld1) late in the IDC. Bottom: DNA content in the parallel samples, with addition of E64 (50 $\mu \mathrm{M})$. Mean of 2 technical replicates. Representative of 2 experiments. i Nuclear centers following PfPP1-DD knockdown at 44 hpi, as in $\mathbf{g}$. Mean \pm s.e.m.; $\mathrm{n}=4$ experiments; two-tailed $t$ test. Scale bar: $5 \mu$ m. j Egress and egress-to-invasion following induction of partial PfPP1-DD knockdown at sublethal doses of Shld1. Mean \pm s.e.m.; $n=4$ experiments; two-tailed $t$ test. Source data are provided as a Source Data file.

form normal rhoptries (antigen PfRhopH3) (Supplementary Fig. $4 \mathrm{e}-\mathrm{g})$.

To initiate egress from erythrocytes at the end of the IDC, parasites secrete the protease PfSUB1 in a regulated fashion from exoneme organelles into the lumen of the parasitophorous vacuole and activate a proteolytic cascade for sequential rupture of the vacuolar (PVM) and host cell membranes ${ }^{29-31}$ (Fig. 2g). To permit assessment of PVM rupture, we tagged the endogenously expressed PVM protein PfEXP2 at the C-terminus with $\mathrm{GFP}^{32}$ in the $p f p p 1-i K O$ background (Supplementary Fig. $4 \mathrm{~h}-\mathrm{j}$ ). Late in the IDC, PfEXP2-GFP in intact PVMs presents intraerythrocytically as a circular label around the parasites or between replicated parasites, while rupture of the PVM can be observed by the appearance of fluorescent membrane fragments in parasites 
a

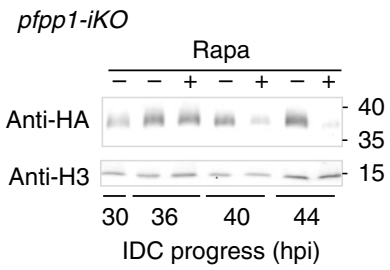

d

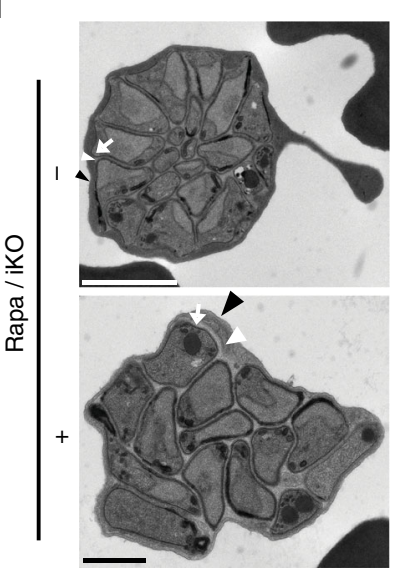

b

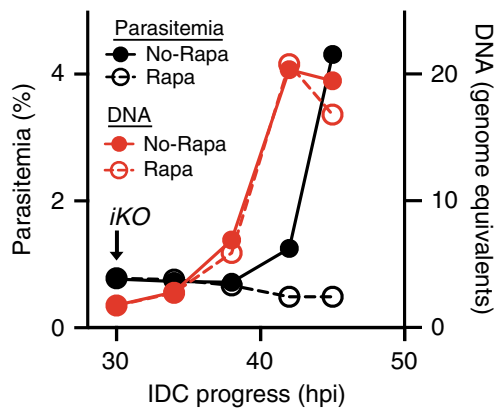

e Phase Hoechst PfMSP1 PfAMA1 Merge

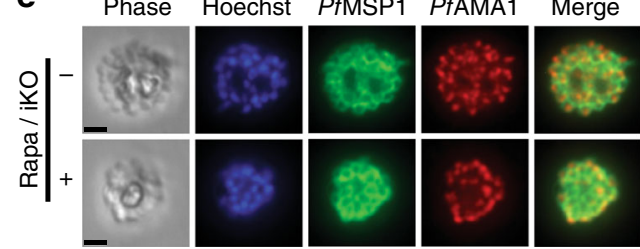

f
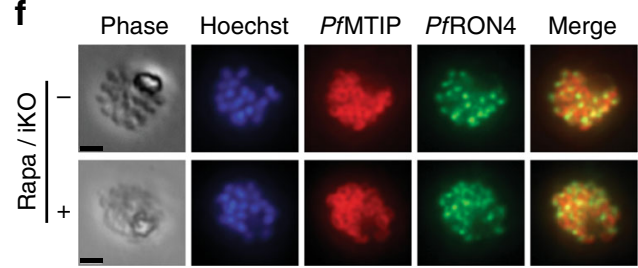

C

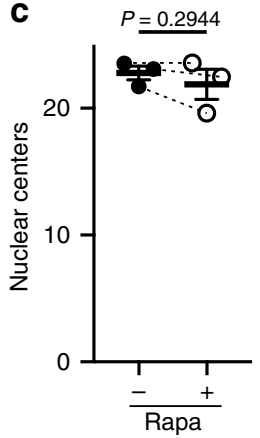

h

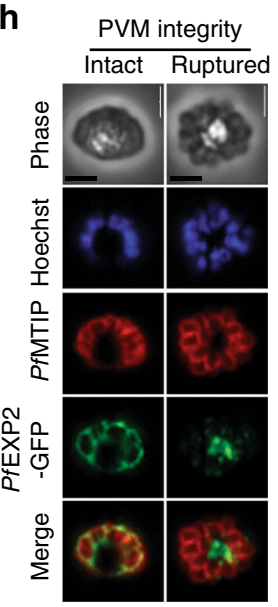

i

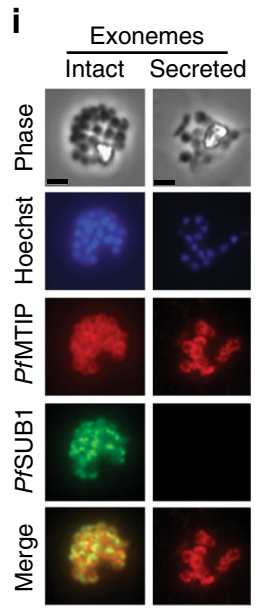

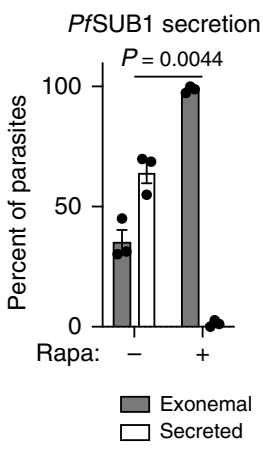

g
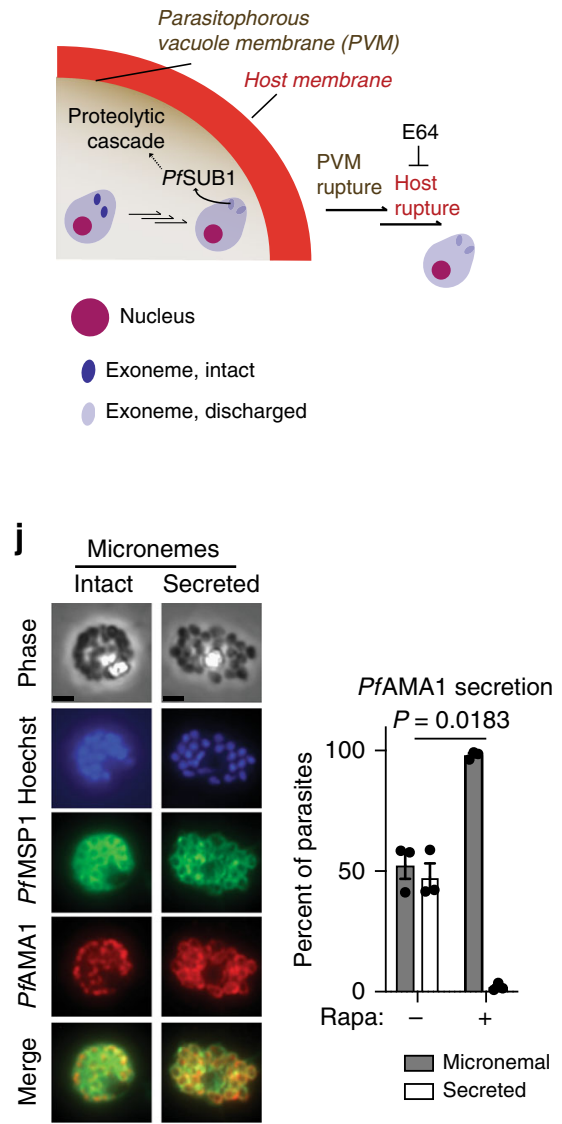

Fig. 2 PfPP1 function at an early step of parasite egress from erythrocytes. a PfPP1-HA 3 expression $+/-$ Rapa-mediated iKO of pfpp1 at 30 hpi, assessed by immunoblot. Representative of 2 experiments. Molecular mass in kDa. b Parasitemia and DNA synthesis following iKO of pfpp1 at 30 hpi, as in Fig. $1 \mathrm{c}$. Mean of 3 technical replicates. Representative of 4 experiments. c Nuclear centers in terminally developed parasites following Rapa-mediated iKO of pfpp 1 at $30 \mathrm{hpi}$, as in Fig. 1. Mean \pm s.e.m.; $n=3$ experiments; two-tailed $t$ test. $\mathbf{d}$ Electron microscopy of terminally developed pfpp1-iKO parasites treated +/-Rapa at 30-hpi. In both images, the different membranes are indicated as follows: erythrocyte (black arrowhead), PV (white arrowhead), and parasite (white arrow). Representative of 2 experiments. Scale bars: $2 \mu \mathrm{m}$ (top), $1 \mu \mathrm{m}$ (bottom). e, f Immunofluorescence analysis of the microneme antigen PfAMA1 e or the rhoptry-neck antigen PfRON4 $\mathbf{f}$ in terminally developed parasites +/-iKO of pfpp1 at 30 hpi. The images also show the parasite plasma membrane marker PfMSP1 e and the inner membrane complex marker PfMTIP f. Scale bar: $2 \mu \mathrm{m}$. For both panels, representative of 2 experiments. $\mathbf{g}$ In a mature parasite, regulated secretion of PFSUB1 from exonemes stimulates a proteolytic cascade leading to sequential rupture of the PVM and the erythrocyte host membranes. h PVM rupture at 45 hpi in pfpp1-iKO / PfEXP2-GFP parasites treated +/- Rapa at 30 hpi. Left: immunofluorescence images of parasites with intact or ruptured PVMs. Right: Proportion of infected cells exhibiting PVM rupture. For $\mathbf{h}-\mathbf{j}$, mean \pm s.e.m.; $n=3$ experiments; two-tailed $t$ test. In $\mathbf{h}-\mathbf{j}$, parasites were treated with $\mathrm{E} 64(50 \mu \mathrm{M})$ at $41 \mathrm{hpi}$; the completion of cytokinesis was assessed with the inner membrane complex marker PfMTIP or the parasite plasma membrane marker PfMSP1. Scale bars (h-j): $2 \mu \mathrm{m}$. i With $+/-$ Rapa-treatment at 30 hpi in pfpp1-iKO parasites, quantification of PfSUB1 secretion from exonemal compartments (loss of punctate fluorescence in images at left), as in $\mathbf{h}$. $\mathbf{j}$ Assessment of PfAMA1 secretion from micronemes, as in $\mathbf{h}$ and $\mathbf{i}$. Source data are provided as a Source Data file. 
treated with E64 to prevent host cell rupture (Fig. $2 \mathrm{~g}, \mathrm{~h})^{32}$. We used labeling by PfEXP2-GFP to test the requirement for PfPP1 in PVM rupture, finding that late induction of iKO blocks the process in virtually all parasites examined (Fig. $2 \mathrm{~h}$ ). We further used immunofluorescence analysis to assess PfSUB1 release required to initiate PVM rupture ${ }^{31}$, finding that rapamycintreated $p f p p 1-i K O$ parasites fail to secrete the protease from exonemes (Fig. 2i). We observed that discharge of micronemes is also blocked by $p f p p 1-i K O$ (Fig. 2j). Our findings indicate an essential function for PfPP1 at an early step of egress, following merozoite development but upstream of discharge of the specialized parasite organelles carrying proteases and other factors utilized for rupture of surrounding membranes.

Identification of PfPP1 substrates by phosphoproteomics. Our reverse genetic analysis establishing the function of PfPP1 at egress maps a factor typically associated with the conventional cell cycle of eukaryotes to the post-replicative stage of the IDC. To test if dephosphorylation of protein substrates accounts for the requirement for PfPP1 in parasites late in the IDC, we implemented a chemical-genetic approach (Fig. 3a) ${ }^{33}$ to measure functional interaction between PfPP1 and calyculin A, an activesite inhibitor of eukaryotic PP1 ${ }^{34}$. We found that knockdown significantly increases the sensitivity of egress-to-invasion of parasites to calyculin A but not to the control antimalarial drug dihydroartemisinin (DHA) (Fig. 3b; Supplementary Fig. 2d), supporting a role for PfPP1 phosphatase activity at this step. We found that PfPP1-mediated intraerythrocytic development is also sensitive to calyculin A (Supplementary Figs. $2 c$ and 5a), suggesting a requirement for protein dephosphorylation during this phase of host cell infection.

To identify potential substrates of PfPP1 and probe regulation of parasites in the late IDC, we carried out a global phosphoproteomic analysis of PfPP1-DD function spanning a period in the IDC from post-replication through egress. Following a restricted period $(1.5 \mathrm{~h})$ of parasite re-invasion from schizont-stage PfPP1-DD parasites into new erythrocytes to generate synchronized cultures, we induced knockdown at $44 \mathrm{hpi}$ and collected samples 4 and $11 \mathrm{~h}$ thereafter (Fig. 3c). We identified a total of 4720 phosphorylation sites from 1170 phosphoproteins, indicating phosphorylation of $\sim 1 / 3$ of all $P$. falciparum proteins we detected in late-stage parasites (Fig. 3d; Supplementary Data 1-3). We detected a comparable or greater number of phosphorylation sites than reported in other phosphoproteomic studies of late-stage $P$. falciparum ${ }^{8,35-38}$. Our dataset thus provides a comprehensive view of phosphorylation events with tight time-resolution through the course of egress. At the earliest timepoint following induction of PfPP1-DD knockdown, there are minimal changes in either the global proteome or phosphoproteome between Shld1-supplemented and knockdown samples, indicating the absence of widespread global changes that may complicate assessment of specific phosphatase functions (Fig. 3d; Supplementary Note 1; Supplementary Fig. 5b-e).

P. falciparum homologs of established PP1 regulators for cell cycle progression ${ }^{9}, 10,39-41$, the nuclear protein sds 22 (in parasites, termed LRR1 for leucine-rich repeat protein $1^{21}$ ) and inhibitor-2 $(\mathrm{I} 2)^{20}$, are among the two most strongly increased factors in protein expression upon PfPP1-knockdown (Fig. 3d). Perturbed expression of these regulators may indicate a conserved function mediated by PfPP1, while an increase in factors for glycolysis and the pentose phosphate pathway identified by gene ontology analysis suggest association with a parasite proliferative state (Supplementary Table 1$)^{42}$. At $48 \mathrm{hpi}$, we observe by both phosphoproteomics and separately by immunoblot analysis increased phosphorylation, by up to $\sim 5$-fold, of Ser-29 of PfHistone H3 (Fig. 3d; Supplementary Fig. 5f), homologous to Ser-28 in the human ortholog studied as a phosphorylation site targeted by $\mathrm{PP}^{43}$. We thus observe among both putative regulators and substrates evidence for conserved PP1 activity in P. falciparum.

PfPP1 regulation of a HECT E3 ligase for egress. The 50 proteins indicated by the 60 phosphopeptides increased $>2$-fold in phosphorylation upon PfPP1-DD knockdown (Fig. 3d, Supplementary Data 3) include chromatin factors (histone H3 variant, histone deacetylase 1 , chromodomain-binding protein), transcription factors from the AP2 family, and vacuolar-proteinsorting family members (VPS11 and VPS18). To focus on potential substrates for essential PfPP1 function at egress, we identified gene products that specifically increase in transcriptional expression late in the IDC (Fig. 3d). The top hit from our phosphoproteomic screen, based on magnitude of increase in phosphorylation with PfPP1-knockdown, is a previously uncharacterized, late IDC-stage protein carrying a 300-amino acid HECT E3 protein-ubiquitin ligase domain at the C-terminus (PF3D7_0628100) (Fig. 3d, e; Supplementary Fig. 5g). In addition to the highly upregulated phosphorylation site (Ser-6138), knockdown of PfPP1-DD also reveals a strongly downregulated site ( $>4$-fold, Tyr-9244); the protein also contains a predicted site for interaction with PfPP $1^{18}$ near the HECT domain (Fig. 3e; Supplementary Fig. 5g). At $>10,000$ amino acids, the protein is the largest in the $P$. falciparum proteome.

The protein, which we name here PfHECT1, is the single HECT domain-containing protein among four in P. falciparum to become increased in expression late in the IDC (Supplementary Fig. 5g). To test for specific HECT activity in parasites late in the IDC, we probed for susceptibility to the small molecule heclin (Fig. 3f). Heclin was identified as a broad-spectrum inhibitor of mammalian HECT enzymes, with biophysical studies suggesting that direct binding by the compound interferes with transfer of ubiquitin from the $\mathrm{E} 2$ adaptor protein to an active-site cysteine in the E3 ligase ${ }^{44}$. In PfPP1-DD parasites stabilized with Shld1, we established the antimalarial activity of heclin toward parasites, with short $(\sim 4 \mathrm{~h})$ and longer periods of exposure $(\sim 24 \mathrm{~h})$ preceding egress exhibiting similar potency of inhibition toward establishment of ring-stage parasites (IC50 20 $\mu \mathrm{M}$ ), suggesting major activity in late schizonts (Fig. 3f; Supplementary Fig. 2d). While PfPP1-DD destabilization does not augment DNA replication defects induced by heclin (Supplementary Figs. 2c and $5 \mathrm{~h}$ ), knockdown in parasites late in the IDC strongly increases susceptibility of egress-to-invasion to the inhibitor, reducing the IC50 by $>100$-fold to submicromolar levels (Fig. 3g; Supplementary Figs. 2d and 5i). An assessment of schizont rupture shows that inhibition of egress by heclin is also strengthened by PfPP1-DD destabilization (Supplementary Figs. 2d and 5j). Phosphoproteomic analysis (Fig. 3d) with chemical-genetic analysis (Fig. $3 \mathrm{~g}$ ) indicates a critical function for PfPP1 in activation of PfHECT1-mediated E3 protein-ubiquitin ligase activity for egress.

PfPP1 regulation of cGMP synthesis. Among other late IDCstage $P$. falciparum genes showing increased phosphorylation with PfPP1-knockdown is a guanylyl cyclase (GC) domaincontaining protein encoded by the gene PF3D7_0381400 (Fig. 3d; Supplementary Fig. 6a), required to synthesize cyclic guanosine monophosphate (cGMP) to stimulate the downstream effector PfProtein Kinase G (PfPKG) and egress from infected ery-

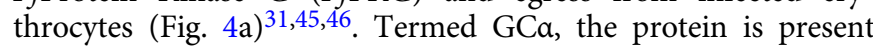
across apicomplexan parasites, and in Toxoplasma gondii is also 
a
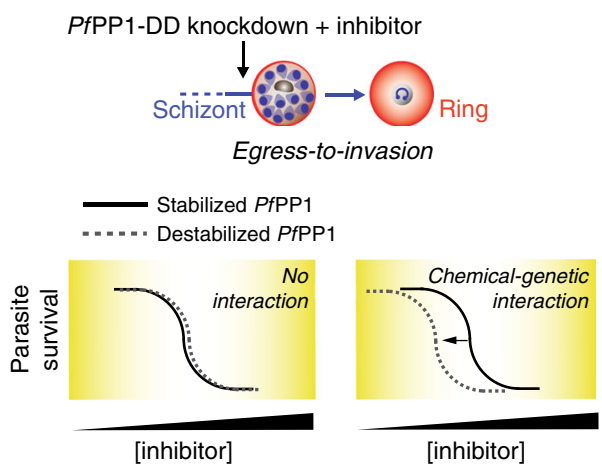

[inhibitor]

d

C

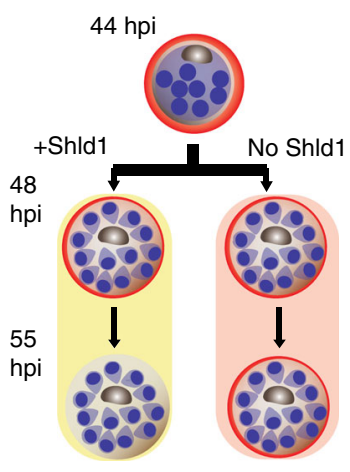

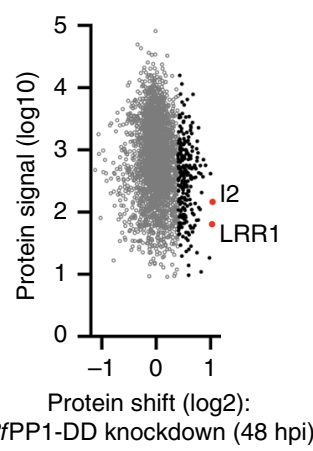

b

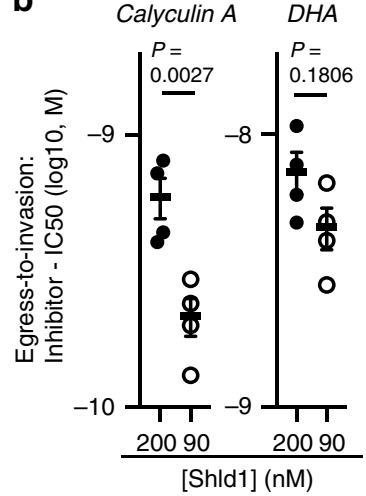

e

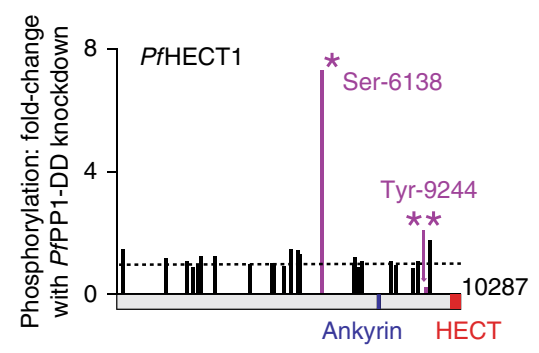

f

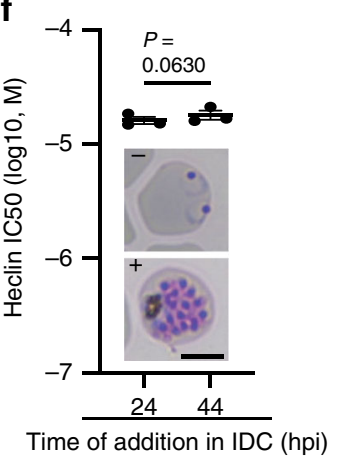

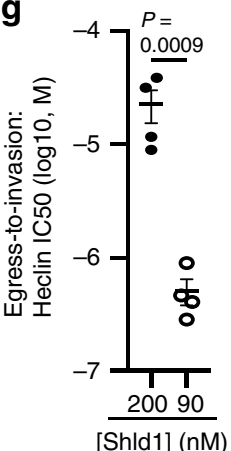

Fig. 3 PfPP1 regulation of a HECT E3 protein-ubiquitin ligase for egress. a Chemical-genetics of PfPP1-DD. We assessed functional interactions between inhibitor-sensitive processes and PfPP1 from shifts in chemical sensitivity induced by knockdown of the phosphatase. b The sensitivities (IC50s) of PfPP1mediated egress-to-invasion to calyculin A and dihydroartemisinin (DHA) at 200 or $90 \mathrm{nM}$ Shld1. Mean \pm s.e.m.; $n=4$ experiments; two-tailed $t$ test. c Scheme for phosphoproteomic analysis of PfPP1-DD knockdown late in the IDC, with samples obtained at 48- and 55-hpi. d Left: At 48 hpi, signal intensities of individual proteins and shifts with PfPP1-knockdown. Pfl2 and PfLRR1 are indicated in red, with the top 5\% of upregulated proteins indicated in black. Right: For all phosphopeptides detected in late-stage parasites, a plot of changes in levels with PfPP1-DD knockdown at 48-hpi ( $y$-axis) versus changes with development from 48 to 55 -hpi in parasites on-Shld1 (x-axis). Thresholds for twofold increased and decreased phosphorylation (log $2=1$, $y$-axis) with knockdown are indicated. Upregulated phosphopeptides from gene products increased in transcription at the schizont-stage ${ }^{16}$ are colored; phosphopeptides in the upper-right quadrant (developmental progression threshold: median value, $x$-axis) least likely to be affected by secondary, developmental-progression defects (Supplementary Note 1) are indicated with filled circles. The phosphorylation site from PfHistone H3 is purple; the sites from PfHECT1 and PfGC $\alpha$ are in red. Representative of 1 experiment. e Schematic of the predicted domains of PfHECT1 protein. We show all phosphosites detected in our study with magnitude of change with PfPP1-DD knockdown at $48 \mathrm{hpi}$ as in $\mathbf{d}$. The most increased (Ser-6138) and decreased phosphorylation sites (Tyr-9244) are indicated with symbols $\left[{ }^{\star}\right]$ and $\left[{ }^{\star \star}\right]$, respectively. $\mathbf{f}$ Sensitivity of PfPP1-DD parasites on-Shld1 (0.5 $\mu$ M) to heclin administered at the midpoint ( $24 \mathrm{hpi}$ ) or late in the IDC ( $44 \mathrm{hpi}$ ), determined from erythrocyte re-invasion. Mean \pm s.e.m.; $n=3$ experiments; two-tailed $t$ test. Representative images of parasites at $55-\mathrm{hpi}+/-$ heclin administration $(100 \mu \mathrm{M})$ at 44 hpi, are shown. Scale bar: $5 \mu \mathrm{m}$. $\mathbf{g}$ The sensitivity of PfPP1mediated egress-to-invasion to heclin, as in $\mathbf{b}$. Mean \pm s.e.m.; $n=4$ experiments; two-tailed $t$ test. Source data are provided as a Source Data file.

essential for egress ${ }^{47-50}$. Given the function we identified for PfPP1 in egress and phosphoregulation of PfGCa, we measured the effect of PfPP1-DD knockdown on cellular cGMP levels, finding that destabilization of the phosphatase enzyme reduces the second messenger in schizont-stage parasites by $\sim 2$-fold
(Fig. 4b). To understand the functional significance of PfPP1 for cGMP-stimulated egress, we implemented chemical-genetics to test for functional interaction with components of signal transduction related to the second messenger. To induce cGMP in parasites late in the IDC, we used zaprinast, a phosphodiesterase 
a

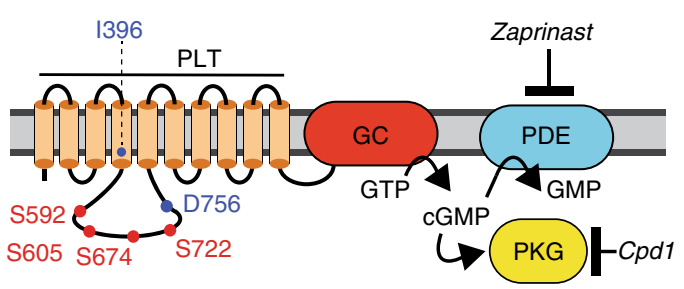

d

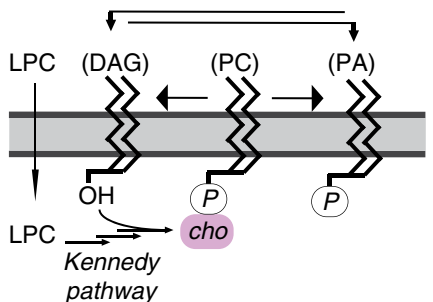

b

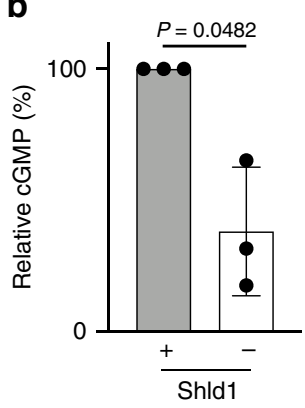

e

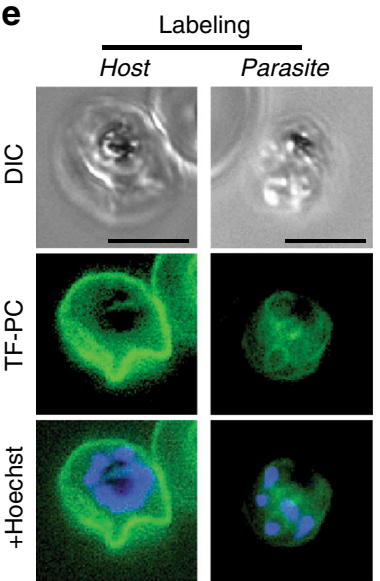

c

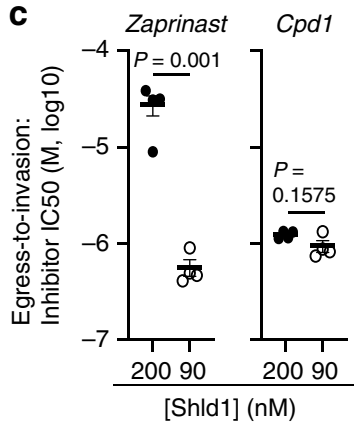

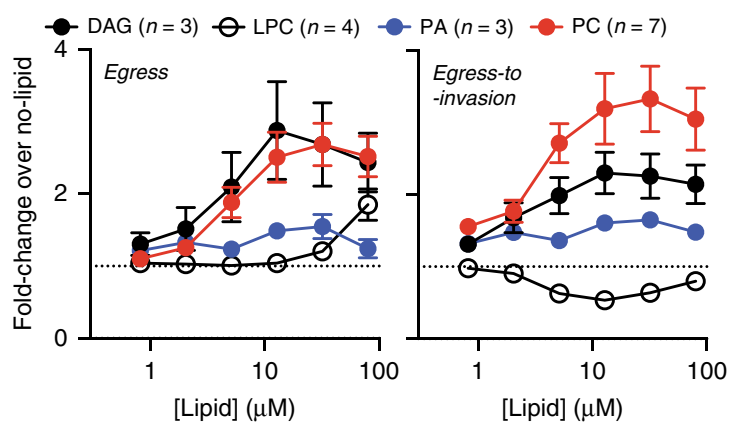

f

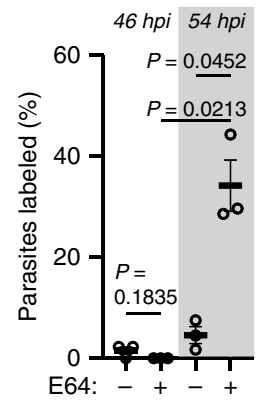

g

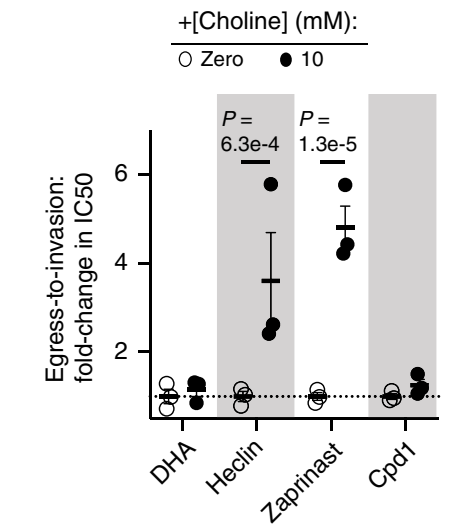

h
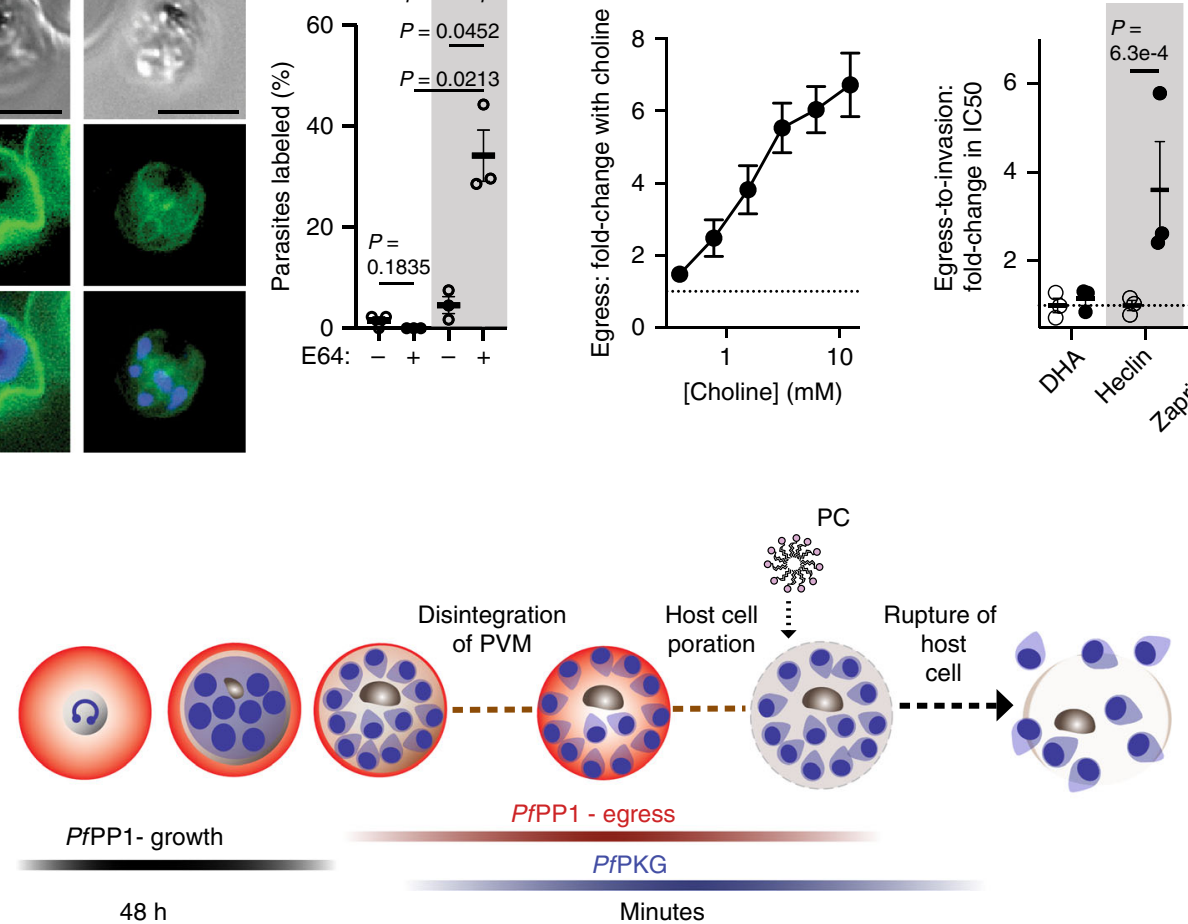

(PDE) inhibitor ${ }^{31,51}$ (Fig. 4a). The susceptibility of egress-toinvasion of PfPP1-DD parasites to zaprinast becomes sharply increased with knockdown: IC50 values drop by as much as $\sim 400-$ fold to submicromolar levels (Fig. 4c; Supplementary Figs. 2d and $6 \mathrm{~b})$. We found that zaprinast inhibition is mitigated by the PfPKG inhibitor Compound-1 (Cpd1) ${ }^{45}$ (Supplementary Figs. 2d and 6c), consistent with the interpretation that toxicity of the PDE inhibitor requires activation of the well-established downstream effector of cGMP signaling in Plasmodium parasites. In contrast to zaprinast, functional interaction of PfPP1-DD with Cpd1 is weak, with measurements of egress-to-invasion showing no chemical-genetic interaction (Fig. 4c). Parasite egress is more sensitive to Cpd1 with PfPP1-DD knockdown, though much less than the shift in sensitivity observed with zaprinast (Supplementary Figs. 2d and 6d). Our analysis of parasites late in the IDC indicates regulation by PfPP1 of guanylyl cyclase activity, upstream of activation of PfPKG for numerous egress and invasion processes ${ }^{37,52}$. We note that in parental, genetically unmodified parasites, Shld1 does not affect sensitivity to calyculin A, heclin, or zaprinast (Supplementary Figs. $2 \mathrm{~d}$ and $6 \mathrm{e}$ ), indicating that the chemical-genetic interactions we have observed in this study with PfPP1-DD knockdown result from impairment of phosphatase function.

Phosphatidylcholine as an extrinsic signal for egress. $\mathrm{N}$-terminal to the GC domain, GCa contains a putative P4-ATPase phospholipid transporter (PLT) domain predicted to translocate 
Fig. 4 PfPP1 regulation of cGMP and signaling by extrinsic phosphatidylcholine. a PfPP1-regulated phosphorylation and cGMP-based signal transduction initiated by PfGC $\alpha$. We indicate PfPP1-regulated sites (red) and signature sequences for phospholipid transporter (PLT) activity (purple), lle-396 and Asp756 (Supplementary Fig. 6a). cGMP-phosphodiesterase (PDE) and PfPKG, as well as inhibitors of both targets, are depicted. b Relative cGMP at 47.5 hpi in PfPP1-DD-intact ( $0.5 \mu \mathrm{M}$ Shld1) or knockdown parasites. Mean \pm s.e.m.; $n=3$ experiments; two-tailed $t$ test. $\mathbf{c}$ The sensitivity of PfPP1-mediated egress-toinvasion to zaprinast (left) or Cpd1 (right). Mean \pm s.e.m.; $n=4$ experiments; two-tailed $t$ test. $\mathbf{d}$ Top: Phospholipids at the parasite plasma membrane with potential routes of interconversion. Extracellular LPC crosses the plasma membrane, providing substrate for endogenous biosynthesis of PC via the Kennedy Pathway 59, 60. Bottom: Stimulation of egress (left) or egress-to-invasion (right) by supplemented lipids in late IDC PfPP1-DD parasites with partial destabilization (100 nM Shld1), expressed in terms of fold-change relative to no-lipid conditions (mean \pm s.e.m., number of experiments indicated in plot).

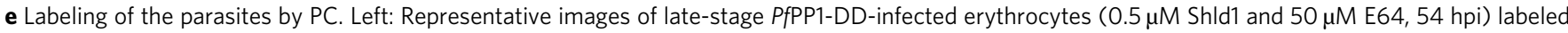
by TF-PC at the erythrocyte membrane or at the plasma membranes of internal parasites. Nuclei are indicated by Hoechst. Scale bar: $5 \mu$ m. Right: The proportion of infected erythrocytes with labeled parasites at the indicated timepoints, $\pm E 64$. Mean \pm s.e.m.; $n=3$ experiments; two-tailed $t$ test.

f Stimulation of PfPP1-mediated egress with supplementation of choline, as in $\mathbf{d}$. Mean \pm s.e.m.; $n=4$ experiments. $\mathbf{g}$ Modulation of PfPP1-regulated cellular processes by supplemented choline. The fold-change in IC50 with additional choline in PfPP1-DD parasites in $150 \mathrm{nM}$ Shld1 is indicated. Mean \pm s.e.m.; $n=$ 3 experiments; multiple two-tailed t tests (false discovery rate, 1\%). h A model for the function of PfPP1. Following regulation of growth during development, PfPP1 is essential for the egress program upstream of cGMP-activated PfPKG and disintegration of the PVM. PC enters host cells following natural permeabilization of the erythrocyte membrane, acting on exposed parasites to stimulate egress. Source data are provided as a Source Data file.

phospholipids from the exoplasmic to the cytoplasmic face of membranes. The fusion of PLT with the GC domain is a structure found only in alveolates ${ }^{53}$, and we observed that PfPP1responsive phosphorylation sites (1.4-2.6-fold upregulation with knockdown) cluster in a parasite-specific cytoplasmic loop of PLT containing a catalytic site predicted essential for P4-ATPase activity (Fig. 4a; Supplementary Fig. 6a; Supplementary Data 3). Mutational analysis in T. gondii GCa indicates the requirement for fusion of the PLT and GC domains as well as ATP-dependent catalysis by the $\mathrm{PLT}^{47,48}$, raising the possibility of a role for phospholipids in function of the protein, potentially for egress. To directly assess involvement of phospholipids in PfPP1-regulated egress, we administered synthetic phospholipids to parasites late in the IDC. We tested phosphatidic acid (PA), known to stimulate host cell egress in $T$. gondii when added extracellularly ${ }^{47}$ and also through endogenously synthesized forms resulting from intracellular phosphorylation of the neutral lipid diacylglycerol $(\mathrm{DAG})^{54,55}$. We also tested phosphatidylcholine (PC), the major species of phospholipid in host serum at concentrations of $\sim 1-2$ $\mathrm{mM}^{56,57}$. In parasites with intact PfPP1-DD (300 nM Shld1), neither phospholipid influences egress (Supplementary Figs. 2d and 6f). In parasites with partially destabilized PfPP1-DD, however, PA stimulates egress by up to $\sim 1.5$-fold and PC up to $\sim 3$ fold, with effects observed as low as $5 \mu \mathrm{M}$ phospholipid (Fig. $4 \mathrm{~d}$; Supplementary Fig. 2d). An effect for PA might be explained by the proposal that the molecule provides one of multiple signals required for productive egress, acting on targets to activate microneme secretion and parasite motility, downstream of cGMP and phosphatidylinositol signaling ${ }^{52,55,58}$. We indeed observed that knockdown of PfPP1-DD late in the IDC increases susceptibility to the DAG-kinase inhibitor R59022 that restricts endogenous PA synthesis (Supplementary Figs. 2d and 6g), consistent with a pro-egress function for the phospholipid in Plasmodium parasites 54 .

A role for PC in egress has not been described. We found that extracellular DAG stimulates egress to similar levels as PC with partial PfPP1-DD destabilization, suggesting convergent targets or efficient conversion between the two lipids upon incorporation into the parasite from the extracellular medium (Fig. 4d; Supplementary Fig. 2d). Merozoites released by PC infect erythrocytes with efficiency comparable to the magnitude of stimulated egress (Fig. 4d; Supplementary Fig. 2d), supporting a physiological function for the phospholipid in invasive parasites near the natural timing for host cell rupture. Compared to PC, DAG yields merozoites with invasiveness reduced by $\sim 30 \%$ (Fig. 4d; Supplementary Fig. 2d). Lysophosphatidylcholine (LPC), which drives parasite PC biosynthesis via the Kennedy
Pathway ${ }^{59,60}$, stimulates egress weakly in comparison to direct administration of the phospholipid (Fig. 4d); and glucose supplementation to increase endogenous $\mathrm{DAG}^{59,61}$ does not stimulate egress (Supplementary Figs. 2d and 6h).

While erythrocyte membranes housing developing $P$. falciparum parasites block access to free $\mathrm{PC}^{59}$, host cells abruptly become permeable to extracellular solutes in the seconds to minutes preceding egress ${ }^{30,32,62}$, presenting a route for direct interaction between parasites and circulating phospholipids. We thus tested accessibility of parasites to PC during egress, using a fluorescent analog (labeled with TopFluor, TF). In most conditions we observed that TF-PC marks only the outer membranes of erythrocytes housing multinucleate schizonts (Fig. 4e). We observed clear labeling of parasites within infected erythrocytes, however, at a later timepoint when parasites have naturally entered the egress program and acquired host cell permeability (Fig. 4e). The intact schizont with permeable erythrocyte membrane is a transient state prior to egress, stabilized by E64-treatment ${ }^{30,62,63}$. We conclude that circulating PC accesses parasites when host cells become permeable to the extracellular environment, shortly before natural host cell rupture.

Endogenous PC synthesis is promoted with the addition of the precursor choline $e^{59,60}$. We found that choline stimulates egress in parasites with partially destabilized PfPP1-DD; albeit in contrast to PC, only at non-physiological serum levels ${ }^{64}$ (Fig. 4f; Supplementary Fig. 2d). Identical, high concentrations of choline do not influence egress by parasites with intact PfPP1-DD (300 nM Shld1) (Supplementary Figs. 2d and 6i). We found that high choline decreases susceptibility of parasites specifically to heclin and zaprinast (Fig. 4g; Supplementary Fig. 2d). Our analysis suggests that a PC signal for egress interacts with cell-intrinsic pathways regulated by PfPP1.

\section{Discussion}

We have functionally characterized PfPP1 through the bloodstage IDC of malaria parasites. In addition to potential conserved roles in development, PfPP1 is essential for egress. At the preerythrocytic liver-stage, analysis by other researchers shows the non-essentiality of Plasmodium PP1 for intrahepatocytic development, though a function for the protein phosphatase in egress into the bloodstream was not assessed 65 . Previous studies of Plasmodium regulation of egress in erythrocytic parasites have focused on the second messenger-responsive protein kinases PfPKG and PfCalcium Dependent Protein Kinase 5 26,31,37,45,66, neither of which is implicated as a substrate of PfPP1 in our study. Our functional analysis of PfPP1 instead delineates a 
regulatory mechanism upstream of stimulation of second messengers. PfPP1 targets a limited set of proteins previously noted as PfPKG-regulated ${ }^{37}$ (Supplementary Data 3), including GCa, raising the possibility of negative feedback for regulation of cGMP synthesis. PfSchizont Egress Antigen $1^{67}$ is among the proteins we found with PfPP1-regulated sites (Supplementary Data 3), perhaps indicating phosphoregulation also of this factor for egress.

We found that PfPP1 regulates PfHECT1 (Supplementary Fig. 7a), demonstrating a role for ubiquitination in egress alongside well-established pathways for phosphorylation and proteolysis. For PfHECT1, PfPP1-mediated phosphorylation in the interior of the polypeptide may indicate a mechanism for regulation of the distal enzymatic domain, e.g. by autoinhibition ${ }^{68}$ or by trans-acting factors ${ }^{69}$ as has been described for other HECT-family E3 ligases.

At multiple stages of the Plasmodium lifecycle, signaling by cGMP is utilized for colonization of new host niches, and studies indicate that a specific timing of activation and level of the second messenger are critical for infectivity $31,70-72$. With the phosphoproteomic analysis, the requirement of PfPP1 at the blood-stage for induction of cellular cGMP in schizont-stage parasites leads us to hypothesize that PfPP1 stimulates synthesis of the cyclic nucleotide by $P f G C a$ (Supplementary Fig. 7a). Regulation of cGMP by PfPP1 may arise from direct interaction of the phosphorylated PLT with the GC domain and/or result from phospholipid translocation regulated by phosphorylation of the PLT. Sensitivity of PfPP1-mediated egress to zaprinast shows that hyperactivation of cGMP synthesis blocks egress, an outcome that we propose reflects a just-in-time regulatory structure controlled by PfPP1. Specifically, we suggest that PfPP1 regulates cGMP induction in coordination with additional processes for egress, with aberrant stimulation of PfPKG preceding other functions resulting in unproductive activation of the egress machinery (Supplementary Fig. 7b).

We discovered PC as a parasite-extrinsic factor that stimulates egress from erythrocytes, distinct from the extracellular LPC signal in P. falciparum that suppresses differentiation to sexualstage forms early in schizogony ${ }^{59}$. This finding supports a model wherein PfPP1 dephosphorylates the phospholipid transporter domain of $P f G C a$ to promote translocation of PC across the parasite plasma membrane and stimulate egress. PC may act as a trigger for cGMP synthesis or as a concurrent signal for egress (Supplementary Fig. 7a). Our study also demonstrates the accessibility of exogenous PC to the host and parasite plasma membranes and ability to influence egress at a late step following host cell poration (Fig. 4h), consistent with function for cGMPactivated PfPKG in free merozoites for erythrocyte invasion ${ }^{37}$. With PfPP1 (Fig. 2), PfPKG is essential also at an earlier step of egress for disruption of the PVM preceding deterioration of the host cell membrane $3^{31,32,63}$. In the absence of host cell permeability at an early stage of egress, intraerythrocytic sources may well provide PC for stimulation of cGMP. Indeed, poration of the PVM preceding PfPKG activation has been reported ${ }^{63}$, indicating a potential source of PC accessible to intracellular merozoites.

Our functional analysis of PfPP1 at egress reveals a regulatory nexus for host cell passage at which cell-intrinsic pathways are coordinated with environmental signals to ensure release of invasive parasites into circulation and infection of new host cells.

\section{Methods}

Reagents and antibodies. Rapamycin (LC laboratories), E64 (Sigma-Aldrich, Cat. No E3132), dihydroartemisinin (Sigma-Aldrich, Cat. No. D7439), calyculin A (Sigma-Aldrich, Cat. No. C5552), heclin (Sigma-Aldrich, Cat. No. SML1396), zaprinast (MP Biomedicals, Cat. No. ICN15693180), were each prepared in DMSO. Choline chloride (Sigma-Aldrich, Cat. No. 7527) was prepared in water. DAG and all phospholipids with mono-unsaturated diacylglycerol backbone $(16: 0,18: 1)$ from Avanti [PA, Cat. No. 840857; PC, Cat. No. 850457; TopFluor-PC, Cat. No. 810281; and DAG, Cat. No. 800815] were solubilized at 1 or $5 \mathrm{mM}$ in $100 \%$ methanol, except PC (water: ethanol: methanol; 1:1:2). LPC (Cat. No. 855675) was solubilized at $200 \mathrm{mM}$ in a 1:1 mixture of ethanol and water. Compound-1 (DMSO-based) was a gift from Dr. Jeffrey Dvorin (Boston Children's Hospital). Dilutions and sources for antibodies for immunoblot or immunofluorescence analysis are as follows: rabbit anti-GAP45 (1:5000, gift from Dr. Julian Rayner, Wellcome Trust Sanger Institute, Hinxton, UK); mouse anti-RhopH3 (1:200, gift from Jean-Francois Dubremetz); rabbit a-MTIP (1:500, gift from Tony Holder, The Francis Crick Institute, UK), mouse $\alpha$-MSP1.19 (1:1000, gift from M. Blackman, The Francis Crick Institute, UK), mouse $\alpha$-RON4 [1:200, home-made ${ }^{73}$ ], mouse $\alpha$-SUB1 (1:2, gift from M. Blackman), rabbit a-AMA1 (1:1000, gift from M. Blackman); rabbit anti-histone H3 (1:10,000, Abcam ab1791); rat anti-HA antibody 3F10 (1:1000, Roche Cat. No. 11867423001); anti-phospho S28 histone H3 antibody (1:1,000, Abcam Cat. No. ab5169); rabbit anti-Pfaldolase-HRP (Abcam, 1:2000); and mouse anti-GFP (Roche, 1:1000). The secondary antibodies used for IFA were Alexa-Fluor 488 and 594-conjugated antibodies against mouse, rat, or rabbit IgG diluted as recommended by the manufacturer (Invitrogen A21208, A11012, A11001, A11008, A21209, and A11005). Shield-1 (Shld1) was synthesized as described ${ }^{25,74}$ and dissolved to $1 \mathrm{mM}$ stock concentration in absolute ethanol before use.

Plasmids. Primers for PCR amplification and verification of transgenesis in $P$. falciparum are shown in Supplementary Table 2. The plasmid for generation of the transgenic parasites $p f p p 1-h a_{3}$ was obtained using the plasmid pLN-PP1-HA 3 -loxP. To generate the plasmid pLN-PP1-HA $-\mathrm{H}_{3}-\mathrm{loxP}$, we first introduced between the BamHI and HpaI sites in pLN-ENR-GFP ${ }^{75}$ a synthetic fragment with sequence for a triple-hemagglutinin tag $\left(\mathrm{HA}_{3}\right)$ followed by a stop codon and a loxP site (IDT DNA), and multiple cloning sites upstream of the tag. The resulting plasmid $\mathrm{pLN}$ $\mathrm{PP} 1-\mathrm{HA}_{3}$-loxP was further modified to target endogenous $p f p p 1$ with $\mathrm{HA}_{3}$ and loxP by introduction of a $5^{\prime}$ homology region for the gene (HR1, 682 bp of genomic DNA sequence for exons 2 and 3 ) fused to a recodonized synthetic fragment (IDT DNA) for exons 4 and 5. The PCR-amplified elements were ligated in a single reaction step by In-Fusion cloning (Clontech) upstream of the $\mathrm{HA}_{3}$ tag in XmaI and AfeI sites of $\mathrm{pLN}-\mathrm{HA}_{3}-\mathrm{loxP}$. The $p f p p 13^{\prime}$ homology region (HR2) carrying $440 \mathrm{bp}$ of the $p f p p 13^{\prime}$-UTR was PCR-amplified and inserted by In-Fusion reaction $3^{\prime}$ of the loxP site between PstI and HpaI. The guide RNA sequence for targeting pfpp1 near exon 3 was cloned into the BbsI sites in pDC2-cam-co-Cas9-U62 hDHFR (gift from Dr. M. Lee, Wellcome Sanger Institute). For subsequent engineering of a $p f p p 1$ conditional knockout in parasites, the pLN-PP1-loxPint plasmid was modified with introduction of the following elements ligated in $5^{\prime}$ to $3^{\prime}$ order between the BamHI and ApaI sites of pLN-ENR-GFP: PCR-amplified fragment of $p f p p 1$ encompassing $5^{\prime}$ UTR and part of exon 1 (382 bp), a synthetic fragment for a recodonized $3^{\prime}$ sequence of exon 1 followed by the artificial loxPint (IDT DNA), and a PCR-amplified fragment of the $5^{\prime}$ end of $p f p p 1$ exon $2(601 \mathrm{bp})$. The plasmid for the guide RNA targeting $p f p p 1$ near exon 1 was constructed as above. To tag endogenous PfEXP2 with GFP, we generated plasmid pLN-PfEXP2-GFP as described $^{32}$. Two homology regions for the gene $p f \exp 2$ were cloned in pLN-ENRGFP on both sides of the GFP coding sequence: 549 bp of $p f \exp 23^{\prime}$ coding sequence without the stop codon in frame with GFP, and $453 \mathrm{bp}$ of $p f \exp 23^{\prime} \mathrm{UTR}$ downstream of GFP. The $p f \exp 2$ guide RNA sequence ${ }^{32}$ was cloned into the BbsI sites of pDC2-cam-co-Cas9-U62-hDHFR. Plasmid pAK8 for 3'-single-crossover HA-DD-tagging at endogenous $p f p p 1$ was constructed in the pJDD41 background ${ }^{26}$ with the PCR-amplified targeting fragment, amplified from $P$. falciparum D10 genomic DNA, ligated between the NotI and XhoI restriction sites. All plasmid sequences were verified before downstream applications. Plasmid pARL2-GFP is previously reported ${ }^{76}$.

Parasite culture. D10 or 3D7 (Walter and Eliza Hall Institute), or p230p-based parasites ${ }^{23}$ were cultured continuously in human erythrocytes ${ }^{77}$ obtained from a commercial source (Research Blood, Boston) or anonymous donors from the French Bloodbank (Etablissement Français du Sang, France) under the approval number 21PLER2016-0103. We complied with all ethical regulations in use of human blood. Continuous culture was typically carried at $2-5 \%$-hematocrit in RPMI-1640 (Sigma Aldrich, Cat. No. R6504) supplemented with HEPES, 25 mM; Albumax II, $4.31 \mathrm{mg} \mathrm{ml}^{-1}$ (Thermo Fisher Scientific), or 10\% human serum; sodium bicarbonate, $2.42 \mathrm{mM}$; and gentamycin $\left(20-25 \mu \mathrm{g} \mathrm{ml}^{-1}\right)$. Parasites were cultured at $37^{\circ} \mathrm{C}$ in hypoxic conditions $\left(1-5 \% \mathrm{O}_{2}, 5 \% \mathrm{CO}_{2}\right.$, with $\mathrm{N}_{2}$ as balance) in modular incubator chambers. Parasites were transfected by electroporation ${ }^{78}$, and treated with WR99210 $(2.5-5 \mathrm{nM})$ or blasticidin $\left(2.5 \mu \mathrm{g} \mathrm{ml}^{-1}\right)$. PfPP1-DD transfectants were further selected for single-crossover integrants by cycles of on-drug and off-drug treatment ${ }^{79,80}$. All transgenic lines were cloned by limiting dilution and genotyped by PCR. Unless otherwise noted, all experiments with PfPP1-DD parasites indicate a line constructed in the D10 background.

We synchronized parasites with heparin $\left(100\right.$ units $\left.\mathrm{ml}^{-1}\right)$ to define restricted periods of invasion ${ }^{81}$. Alternatively, we isolated schizonts by magnetic affinity purification (MACs LS column, Miltenyi, fitted with a 23-gauge needle) or 70\% Percoll cushion, and allowed invasion into uninfected erythrocytes for a defined period. Following invasion, we either added heparin to block further invasion, selectively lysed remaining schizonts by sorbitol treatment $(5 \% \mathrm{w} / \mathrm{v}$ in double- 
distilled water), or isolated recently invaded rings from unruptured schizonts by magnetic affinity purification (MACs LS with 27-gauge needle).

Statistical significance testing. We carried out all tests for statistical significance in Prism software (GraphPad). Unless otherwise stated, $P$ values indicate the results of two-tailed, paired $t$ tests. For multiple $t$ tests, false discovery rate (FDR) calculation was implemented using the default two-stage step-up method of Benjamini, Krieger, and Yekutieli.

Induction of PfPP1-phenotypes through conditional expression. For assays, we induced parasites for either knockout of $p f p p 1$ [mock- (DMSO) versus Rapa, 10 $\mathrm{nM}$ ] or knockdown of PfPP1-DD [Shld1 $(0.2-0.5 \mu \mathrm{M})$ versus ethanol vehicle]. In knockout parasites, we washed away Rapa $4 \mathrm{~h}$ following addition.

Immunoblot analysis. For immunoblot analysis, we released parasites from ery throcytes with cold PBS containing $0.1-0.2 \%$ saponin, and boiled in SDS-PAGE sample buffer ${ }^{80}$. Following electrophoretic separation, proteins were transferred to a nitrocellulose membrane, and immunoblot analysis was carried out using the LICOR system (Lincoln, USA), or the Chemidoc system (Bio-Rad).

Microscopy. For quantitative assessment of nuclear centers in terminally developed PfPP1-DD parasites [ $\sim 54-60 \mathrm{hpi} ;+\mathrm{E} 64(50 \mu \mathrm{M})$ since $\sim 45 \mathrm{hpi}]$, we collected $\sim 500,000$ infected cells onto glass slides by cytospin centrifugation, followed by fixation in methanol and staining with May-Grünwald-Giemsa. All infected cells encountered in visual fields by conventional light microscopy ( $>54$ per sample) were counted. In the $p f p p 1-i K O$ line, we used immunofluorescence microscopy (see below) to identify segmented parasites that stained positive for the antigen PfMTIP before counting nuclei stained with DAPI. For immunofluorescence ${ }^{66}$, thin smears were fixed in 4\%-paraformaldehyde in PBS for $10 \mathrm{~min}$ at room temperature or overnight at $4{ }^{\circ} \mathrm{C}$ in a humidified chamber followed by extensive washing in buffer; permeabilized with $0.1 \%$-Triton-X-100/PBS for 10 minutes at room temperature before further washing; blocked with $3 \%$ BSA/PBS for $>1 \mathrm{~h}$ at room temperature or overnight at $4{ }^{\circ} \mathrm{C}$; treated with primary antibody overnight at $4{ }^{\circ} \mathrm{C}$; washed extensively before treatment with the appropriate Alexa-Fluor 488 or 594conjugated secondary antibody for $1 \mathrm{~h}$ at room temperature; washed and prepared in DAPI-containing mounting solution for imaging. Images were taken with a Zeiss Observer Z1, Zeiss Axioimager Z2, or confocal Zeiss LSM880 equipped with an Airyscan module, and processed with Zen 2 blue edition software (Zeiss) or $\mathrm{Fiji}^{82}$. For immunofluorescence assays for PVM rupture or secretion of exoneme or microneme antigens, parasites were treated at 41 hpi with $50 \mu \mathrm{M}$ E64 and smeared $4-5 \mathrm{~h}$ later for analysis.

For assessment of TF-PC labeling of $P$. falciparum, we treated synchronous $( \pm 1$ h) PfPP1-DD parasites $(+0.5 \mu \mathrm{M}$ Shld1) with or without E64 $(50 \mu \mathrm{M})$ for $\sim 7 \mathrm{~h}$ before image acquisition at the indicated timepoints. After evaporation of TF-PC on the surface of multiplate wells, we added parasites in standard media $(2 \%$ hematocrit) for a final concentration of fluorescent label of $100 \mu \mathrm{M}$. After $\sim 30 \mathrm{~min}$ at $37^{\circ} \mathrm{C}$ in standard culture conditions, cells were collected and stored at $4{ }^{\circ} \mathrm{C}$ until imaging carried out over the course of the next $\sim 2 \mathrm{~h}$. Just before imaging, parasites were spotted and mixed with Hoechst dye on coverslips (No. 1.5) pre-treated with Concanavalin-A (Sigma-Aldrich, Cat. No. C5275, $0.5 \mathrm{mg} \mathrm{ml}^{-1}$ in PBS; spread and dried at $37^{\circ} \mathrm{C}$ for $\sim 30 \mathrm{~min}$ ) immediately before sealing by surface tension and dispersion of cell suspension with a glass slide. Images were acquired with a $63 \times-$ objective on the Zeiss Observer Z1, in the DIC, DAPI, and GFP channels. We scored 40-66 multinucleate, infected cells per condition to estimate internal labeling of parasites.

For transmission electron microscopy (TEM) of $p f p p 1-i K O$ cells, we directly added $25 \%$ glutaraldehyde (EM grade) to the culture medium to obtain a final concentration of $2.5 \%$. After 10 min incubation at room temperature, we centrifuged the cells and resuspended the pellet in 20 volumes of cacodylate buffer $(0.1 \mathrm{M})$ containing $2.5 \%$ glutaraldehyde and $5 \mathrm{mM} \mathrm{CaCl}_{2}$.The suspension was left 2 hours at RT before long-term storage at $4{ }^{\circ} \mathrm{C}$ in fixative until further processing. All the following incubation steps were performed in suspension, followed by centrifugation using a benchtop microcentrifuge. Cells were washed with cacodylate buffer and post-fixed with $1 \% \mathrm{O}_{\mathrm{s}} \mathrm{O}_{4}$ and $1.5 \%$ potassium ferrocyanide in cacodylate buffer for $1 \mathrm{~h}$. After washing with distilled water, samples were incubated overnight in $2 \%$ uranyl acetate in water and dehydrated in graded series of acetonitrile. Impregnation in Epon 812 was performed in suspension on a rotary shaker for $1 \mathrm{~h}$ in Epon: acetonitrile (1:1) and $2 \times 1 \mathrm{~h}$ in 100\% Epon. After the last step, cells were pelleted in fresh epon and polymerized for $48 \mathrm{~h}$ at $60^{\circ} \mathrm{C} .70 \mathrm{~nm}$ sections were made with an ultramicrotome Leica UC7, contrasted with uranyl acetate and lead citrate and imaged by TEM on a JEOL 1200 EX. All chemicals were purchased from Electron Microscopy Sciences (USA).

TEM analysis of PfPP1-DD parasites was carried out similarly, with some modifications. For fixation, 1 volume of suspended culture $(>\sim 5 \mu$ l packed cell volume) was supplemented with 1 volume of a $2 \mathrm{x}$ fixative solution $(5 \%$ glutaraldehyde, $2.5 \%$ paraformaldehyde, $0.06 \%$ picric acid in $0.2 \mathrm{M}$ cacodylate buffer, $\mathrm{pH}$ 7.4), spun briefly at $500 \mathrm{~g}$, and stored at $4{ }^{\circ} \mathrm{C}$ before further processing. Following fixation, cells were washed in water, then maleate buffer before incubation in $2 \%$ uranyl acetate $(1 \mathrm{~h})$. Following washes in water, dehydration was done in grades of alcohol (10 min each at 50\%, 70\%, 90\%, and $2 \times 100 \%)$. The samples were then put in propyleneoxide for $1 \mathrm{~h}$ and infiltrated overnight in a 1:1 mixture of propyleneoxide and TAAB Epon (Marivac Canada Inc. St. Laurent, Canada). The following day, the samples were embedded in TAAB Epon and polymerized at $60^{\circ} \mathrm{C}$ for $48 \mathrm{~h}$. Ultrathin sections (about $60 \mathrm{~nm}$ ) were cut on a Reichert Ultracut-S microtome, picked up on to copper grids stained with lead citrate and examined in a JEOL $1200 \mathrm{EX}$ or a TecnaiG ${ }^{2}$ Spirit BioTWIN. Images were recorded with an AMT 2k CCD camera.

Flow-cytometry and analysis. All measurements of parasitemia by flowcytometry were carried out with staining of fixed cells with SYBR-Green I (Invitrogen) to distinguish DNA-containing parasitized erythrocytes from uninfected, enucleate erythrocytes ${ }^{80,83-85}$. Fixation of PfPP1-DD cell suspension was carried out by addition of $>3$ volumes of PBS supplemented with paraformaldehyde $(4 \%$ final concentration) and glutaraldehyde (0.0075-0.015\% final concentration), followed by storage at $4{ }^{\circ} \mathrm{C}$ for $>12 \mathrm{~h}$ before further washes in buffer. For quantitative measurements of cellular DNA, fixed cells were permeabilized with Triton-X-100 $(0.1 \%)$ and RNase-treated $\left(\sim 0.3 \mathrm{mg} \mathrm{ml}^{-1}\right)$ before staining ${ }^{85}$. For $p f p p 1-i K O$ parasites, cells were fixed by addition of an equal volume of PBS-paraformaldehyde (8\%); fluorescence was measured with a BD FACS Canto I cytometer and 100,000 events were recorded per sample. For PfPP1-DD parasites, flow-cytometry was carried out with a MacsQUANT 10 instrument (Miltenyi) on the FITC channel. We measured at least 20,000 cells per sample. All flow-cytometry data were analyzed with FlowJo software.

To calculate cellular DNA content, we used fluorescence measurements from uninfected erythrocytes (zero genomes), singly-infected rings (1 genome), and doubly-infected rings ( 2 genomes), to generate standard curves for translation of total fluorescence of an infected erythrocyte population into genome equivalents (Supplementary Fig. 2). In some measurements, we applied a background correction across all samples to subtract the contribution of parasite cells that did not advance into DNA replication by 48 -hpi (i.e. in +Shld1-conditions). To calculate egress from parasites late in the IDC in an experiment, we used the remaining schizont populations measured at the end of an assay. We treated schizont levels in No-Shld1 conditions (or $50 \mathrm{nM}$ Shld1, Supplementary Fig. 6f, i) as a measure of no-egress (zero), and levels with high-Shld1 as full egress (100\%). We similarly calculated egress-to-invasion from parasites late in the IDC using ring-stage parasite levels. Fold-change in egress, as reported in Fig. 4, is the quotient of schizont levels at the end of the assay in the absence of chemical (numerator) to schizonts levels left in the presence of chemical (denominator).

Measurement of cellular cGMP. PfPP1-DD parasites $(0.5 \mu \mathrm{M}$ Shld1) were synchronized by magnetic affinity purification of schizonts followed by heparin treatment to define an invasion window of $4 \mathrm{~h}$ (see above). At $44 \mathrm{hpi}( \pm 2 \mathrm{~h})$, we repeated magnetic affinity purification in the absence of Shldl, using a $27 \mathrm{G}$ insulin needle for maximum recovery of schizonts. Following four washes in excess volume of RPMI to remove residual Shld1, schizonts were resuspended in media $(\sim 3.5$ $-6.5 \times 10^{6}$ cells $\mathrm{ml}^{-1}$ ), split with either $0.5 \mu \mathrm{M}$ Shldl or ethanol vehicle, and replaced in culture at 2 technical replicates per experimental condition. After $3.5 \mathrm{~h}$, cells were centrifuged twice $(930 \times g, 2 \mathrm{~min}$, room temperature) to extensively remove culture supernatant before snap-freezing in liquid nitrogen and long-term storage at $-80^{\circ} \mathrm{C}$.

We used a competitive ELISA assay kit (Cayman Chemical Cat. No. 581021) to measure cGMP extracted from schizonts. We followed the manufacturer's acetylation protocol following direct lysis of cells in $0.1 \mathrm{M} \mathrm{HCl}$ and appropriate dilutions into the commercial ELISA buffer. Following 14-18 h of incubation of plated samples with cGMP antiserum and tracer, and several washes, we carried out development with the colorometric Ellmans Reagent for $\sim 1 \mathrm{~h}$ before absorbance measurements $(\lambda, 405)$. Reported data are from measurements of samples corresponding to approximately $0.7-5 \times 10^{6}$ schizonts (average of two technical replicates per experiment). Sample cGMP levels are estimated from logit transformed linear fits of measurements of provided cGMP standards.

\section{Proteomic and phosphoproteomic profiling. PfPP1-DD parasites were syn-} chronized as described above with MACs purification of schizonts and treatment with sorbitol ( $5 \% \mathrm{w} / \mathrm{v}$ in water) after 1.5 hours of invasion into uninfected erythrocytes to eliminate remaining schizonts and isolate freshly re-invaded rings. Approximately $10 \times 10^{10}$ synchronous ring-stage PfPP1-HA-DD parasites in Shld1 $(0.3 \mu \mathrm{M})$ were cultured to $44 \mathrm{hpi}$ with a preceding change of warm media at $\sim 24$ hpi, washed extensively and replated in warm $\left(37^{\circ} \mathrm{C}\right)$ complete-RPMI at $\sim 5 \times 10^{6}$ parasitized erythrocytes per ml of culture $\pm 0.3 \mu \mathrm{M}$ Shldl. At $48 \mathrm{hpi}$, for each Shld 1 condition, 2 technical replicates each with or without Shld $1\left(\sim 1 \times 10^{10}\right.$ parasitized erythrocytes per replicate) were centrifuged at room temperature $(500 \mathrm{~g})$, and the pellet was frozen at $-80^{\circ} \mathrm{C}$ for downstream processing. Remaining cultures $(3$ technical replicates, each with or without Shld1) were supplemented with E64 $(15 \mu \mathrm{M})$ for further culture until $55 \mathrm{hpi}$ for collection as described above.

Further protein extraction steps for each of the ten samples were carried out in solutions supplemented with cOmplete protease inhibitors (Roche) and PhosSTOP phosphatase inhibitor cocktail (Roche). We released parasites with $0.05 \%$ saponin in ice-cold PBS administered over several washes at $4{ }^{\circ} \mathrm{C}$, for a total of $\sim 6.5$ volume 
of buffer for 1 packed erythrocyte volume (PEV) of frozen pellet. Following additional washes in ice-cold PBS without saponin, we added $>1$ original PEV of 8 $\mathrm{M}$ urea lysis buffer $(100 \mathrm{mM} \mathrm{NaCl}, 25 \mathrm{mM}$ Tris- $\mathrm{HCl} / \mathrm{pH} 8)$, and subjected each sample to $5 \times$ freeze $\left(-80^{\circ} \mathrm{C}\right)$-thaw cycles before centrifugation at room temperature to separate protein-containing supernatant from pelleted cellular debris. Yields for each of the 10 samples ranged from $5-7 \mathrm{mg}$ as assessed with the Pierce BCA (Bicinchoninic acid) protein assay. We reduced disulfide bonds with 5 $\mathrm{mM}$ tris(2-chloroethyl) phosphate (TCEP) for $30 \mathrm{~min}$ at room temperature, alkylated cysteines with $15 \mathrm{mM}$ iodoacetamide for $30 \mathrm{~min}$ at room temperature in the dark, and quenched excess iodoacetamide by treatment with $5 \mathrm{mM}$ dithiothreitol (DTT) for $15 \mathrm{~min}$ at room temperature. We precipitated protein in chloroform-methanol ${ }^{86}$ before resuspension ( $8 \mathrm{M}$ urea, $50 \mathrm{mM}$ HEPES pH 8.5$)$ and before dilution of urea to $1 \mathrm{M}(50 \mathrm{mM}$ HEPES $\mathrm{pH} 8.5)$ for digestion with LysC protease $\left(1: 100\right.$ protease-to-protein ratio, $3 \mathrm{~h}$ at $\left.37^{\circ} \mathrm{C}\right)$ before the addition of trypsin (1:100 protease-to-protein ratio) and continued digestion overnight at $37^{\circ} \mathrm{C}$. We quenched the reaction with $1 \%$ formic acid, carried out C18 solid-phase extraction (Sep-Pak, Waters), and precipitated peptides with vacuum centrifugation.

To perform the isobaric labeling with tandem mass tags (TMTs), $200 \mu \mathrm{g}$ of peptides from each sample was dissolved in Buffer 1 (100 mM HEPES, pH 8.5). We carried out labeling with TMT reagents ${ }^{87}$, according to manufacturer instructions (Thermo-Fisher Scientific). Following combination of the 10 TMT-labeled samples with matched protein mass between samples, the mixture was vacuum-centrifuged and subjected to C18 solid-phase extraction (Sep-Pak, Waters) and eluate was collected.

Peptides were resuspended in Buffer 1, followed by enrichment of phosphopeptides with High-Select ${ }^{\text {tu }}$ Fe-NTA Phosphopeptide Enrichment Kit (Thermo-Scientific Cat. No. A32992) ${ }^{88}$. The flow-through was retained for analysis of the proteome. Peptides and enriched phosphopeptides were dried by vacuum centrifugation.

For proteomic analysis, the TMT-labeled peptide pool was fractionated via basic $\mathrm{pH}$ reversed-phase (BPRP) high-performance liquid chromatography ${ }^{87}$. Eluted fractions were desalted, dried by vacuum centrifugation, and resuspended in a solution of $5 \%$ acetonitrile and $5 \%$ formic acid, for mass spectrometry-based measurements.

For phosphopeptide analysis, we used the Pierce Off-line BPRP fractionation kit (Thermo Scientific), collecting and processing fractions for LC-MS/MS-based analysis $^{85}$.

We collected MS/MS data using an Orbitrap Fusion mass spectrometer (Thermo Fisher Scientific) coupled to a Proxeon EASY-nLC 1000 liquid chromatography (LC) pump (Thermo Fisher Scientific). For each analysis, $1 \mu \mathrm{g}$ protein was loaded onto the LC onto an in-house pulled C18 column $[30-35 \mathrm{~cm}$, 2.6 um Accucore (Thermo Fisher), 100 um ID] for MS-analysis ${ }^{85}$.

Global proteome and phosphoproteome analyses each employed the multinotch MS3-based method ${ }^{85,89}$. Global proteome and phosphoproteome analyses used an MS3-based TMT method ${ }^{90,91}$, which has been shown to reduce ion interference compared to MS2 quantification ${ }^{92}$.

Mass spectra were analyzed with a SEQUEST-based pipeline ${ }^{85,93}$. Peptide spectral matches (PSMs) were carried out a $1 \%$ FDR, and filtered ${ }^{85}$. To quantify the TMT-reporter ion, in each channel ( $0.003 \mathrm{Th}$ range to distinguish reporters) we extracted the summed signal-to-noise ratio and found the closest matching centroid to the expected mass. For proteomic analysis, PSMs (1\% FDR) were collapsed to whole proteins ( $1 \%$ FDR). We used principles of parsimony to assemble the protein set, aiming to identify the smallest number of distinct polypeptides required to explain the observed PSMs. Relative protein levels were quantified by calculating the sum of reporter ion counts across associated PSMs ${ }^{33}$. MS3 spectra represented in $<2$ TMT channels, and MS3 spectra with TMT reporter summed spectra of $<100$, or no MS3 spectra, were not considered for further analysis ${ }^{89}$. Protein quantitation values were exported to Microsoft Excel. To normalize for variations in sample loading, within each TMT reporter ion channel, each parasite protein was normalized to the total signal of the $P$. falciparum proteome measured in that channel ${ }^{85}$. For phosphoproteomics, the signal intensity of each phosphopeptide in a single TMT channel was normalized to the level of the parent protein in the same experimental condition (average of normalized values for technical replicate measurements from proteomics). We did not apply this adjustment for the $<2 \%$ of phosphopeptides that could not be mapped to a parent protein in proteomics. Raw signal intensities for non-phosphorylated peptides detected in phosphopeptide-enriched samples are reported in the Source Data (sheet: non-phos peptides), with column headings for the table as in Supplementary Data 3. For analysis in Supplementary Fig. 5d, non-phosphorylated peptide levels were normalized according to parent protein levels as described above for phosphopeptides.

For the 19 proteins shown to be increased in expression ( $>1.3$-fold) in two proteomics measurements from separate experiments (Supplementary Data 1 and 2 ), we carried out gene ontology enrichment analysis using the webtool at PlasmoDB (http://www.plasmodb.org). The ten most enriched GO categories are shown in Supplementary Table 1.

For restriction of candidate substrates of PfPP1 to proteins expressed late in the IDC, we used web-based tools at PlasmoDB (https://www.plasmodb.org) relying on published transcriptome data ${ }^{16}$ to identify $P$. falciparum genes that are upregulated at the 40- or 48 hpi-mark of the IDC by at least threefold over levels at the midpoint of the IDC (average expression of 16 and $24 \mathrm{hpi}$ ), and by at least 1.5 -fold over levels at $32 \mathrm{hpi}$

Chemical-genetic assays. Compounds in DMSO (0.1-10 mM) were printed onto the surface of standard 96-well plates using a D300e automated dispenser (Hewlett Packard), and stored at $-20^{\circ} \mathrm{C}$ until addition of parasites. Chemicals in water were added individually onto the surface of 96 -well plates, or added directly at the high concentration to parasites in wells before serial dilution in-plate. Phospholipids (or methanol vehicle) at 10x final concentration were added to the surface of plates and allowed to evaporate before addition of parasites. Synchronized PfPP1-DD parasites were washed extensively in media without Shld1 and supplemented with varying concentrations of Shld1 (constant volume of ethanol carrier across doses), as indicated in the data. Parasites with Shld1, set at $0.5 \%$-hematocrit, were added at $100 \mu \mathrm{l}$ volume to wells with compounds to attain the reported final concentrations of inhibitors. Wells immediately surrounding the samples were filled with water or aqueous solution to prevent evaporation through the course of the assay. Parasites were allowed to incubate with inhibitor for $\sim 20 \mathrm{~h}$ before fixation and measurement of egress from schizonts and reinvasion to rings by flow-cytometry.

For measurements in Supplementary Fig. $5 \mathrm{a}, 1 \mu \mathrm{l}$ of calyculin $(0.1 \mathrm{mM})$ was diluted to a final concentration of $500 \mathrm{nM}$ directly into PfPP1-DD parasites $(0.3 \%$ hematocrit) with or without $0.5 \mu \mathrm{M}$ Shld1 before manual serial dilution (fivefold) in 96-well plates.

Reporting summary. Further information on research design is available in the Nature Research Reporting Summary linked to this article.

\section{Data availability}

The mass spectrometry proteomics data have been deposited to the ProteomeXchange Consortium (http://proteomecentral.proteomexchange.org) via the PRIDE partner repository ${ }^{94}$ with the dataset identifier PXD018718 and DOI 10.6019/PXD018718. All data generated or analyzed during this study are included in this published article (and its supplementary information files). A reporting summary for this article is available as a Supplementary Information file. Source data are provided with this paper.

Received: 4 December 2019; Accepted: 19 June 2020; Published online: 15 July 2020

\section{References}

1. Gerald, N., Mahajan, B. \& Kumar, S. Mitosis in the human malaria parasite Plasmodium falciparum. Eukaryot. Cell 10, 474-482 (2011).

2. Arnot, D. E., Ronander, E. \& Bengtsson, D. C. The progression of the intraerythrocytic cell cycle of Plasmodium falciparum and the role of the centriolar plaques in asynchronous mitotic division during schizogony. Int. J. Parasitol. 41, 71-80 (2011).

3. Pease, B. N. et al. Global analysis of protein expression and phosphorylation of three stages of Plasmodium falciparum intraerythrocytic development. J. Proteome Res. 12, 4028-4045 (2013).

4. Bushell, E. et al. Functional profiling of a Plasmodium genome reveals an abundance of essential genes. Cell 170, 260-272 (2017).

5. Zhang, M. et al. Uncovering the essential genes of the human malaria parasite Plasmodium falciparum by saturation mutagenesis. Science 360, eaap7847-30 (2018)

6. Tewari, R. et al. The systematic functional analysis of Plasmodium protein kinases identifies essential regulators of mosquito transmission. Cell Host Microbe 8, 377-387 (2010).

7. Guttery, D. S. et al. Genome-wide functional analysis of Plasmodium protein phosphatases reveals key regulators of parasite development and differentiation. Cell Host Microbe 16, 128-140 (2014).

8. Solyakov, L. et al. Global kinomic and phospho-proteomic analyses of the human malaria parasite Plasmodium falciparum. Nat. Commun. 2, 565 (2011)

9. Bollen, M., Gerlich, D. W. \& Lesage, B. Mitotic phosphatases: from entry guards to exit guides. Trends Cell Biol. 19, 531-541 (2009).

10. Rodrigues, N. T. L. et al. Kinetochore-localized PP1-Sds22 couples chromosome segregation to polar relaxation. Nature 524, 489-492 (2015).

11. Ohkura, H., Kinoshita, N., Miyatani, S., Toda, T. \& Yanagida, M. The fission yeast dis $2+$ gene required for chromosome disjoining encodes one of two putative type 1 protein phosphatases. Cell 57, 997-1007 (1989).

12. Ceulemans, H. \& Bollen, M. Functional diversity of protein phosphatase-1, a cellular economizer and reset button. Physiolog. Rev. 84, 1-39 (2004).

13. Cohen, P., Schelling, D. L. \& Stark, M. J. Remarkable similarities between yeast and mammalian protein phosphatases. FEBS Lett. 250, 601-606 (1989).

14. Kumar, R., Adams, B., Oldenburg, A., Musiyenko, A. \& Barik, S. Characterisation and expression of a PP1 serine/threonine protein phosphatase (PfPP1) from the malaria parasite, Plasmodium falciparum: 
demonstration of its essential role using RNA interference. Malar. J. 1, 5 (2002).

15. Bártfai, R. et al. H2A.Z demarcates intergenic regions of the Plasmodium falciparum epigenome that are dynamically marked by $\mathrm{H} 3 \mathrm{~K} 9 \mathrm{ac}$ and H3K4me3. PLoS Pathog. 6, e1001223-14 (2010).

16. Otto, T. D. et al. New insights into the blood-stage transcriptome of Plasmodium falciparum using RNA-Seq. Mol. Microbiol. 76, 12-24 (2010).

17. Gnangnon, B. et al. Plasmodium pseudo-tyrosine kinase-like binds PP1 and SERA5 and is exported to host erythrocytes. Sci. Rep. 9, 8120 (2019).

18. Hollin, T., De Witte, C., Lenne, A., Pierrot, C. \& Khalife, J. Analysis of the interactome of the Ser/Thr Protein Phosphatase type 1 in Plasmodium falciparum. BMC Genomics 17, 246 (2016).

19. Hollin, T. et al. Essential role of GEXP15, a specific Protein Phosphatase type 1 partner, in Plasmodium berghei in asexual erythrocytic proliferation and transmission. PLoS Pathog. 15, e1007973-32 (2019).

20. Fréville, A. et al. Plasmodium falciparum encodes a conserved active inhibitor2 for Protein Phosphatase type 1: perspectives for novel anti-plasmodial therapy. BMC Biol. 11, 80 (2013).

21. Daher, W. et al. Regulation of protein phosphatase type 1 and cell cycle progression by PfLRR1, a novel leucine-rich repeat protein of the human malaria parasite Plasmodium falciparum. Mol. Microbiol. 60, 578-590 (2006).

22. Bloecher, A. \& Tatchell, K. Dynamic localization of protein phosphatase type 1 in the mitotic cell cycle of Saccharomyces cerevisiae. J. Cell Biol. 149, $125-140$ (2000).

23. Knuepfer, E., Napiorkowska, M., van Ooij, C. \& Holder, A. A. Generating conditional gene knockouts in Plasmodium - a toolkit to produce stable DiCre recombinase-expressing parasite lines using CRISPR/Cas9. Sci. Rep. 7, 3881 (2017).

24. Collins, C. R. et al. Robust inducible Cre recombinase activity in the human malaria parasite Plasmodium falciparumenables efficient gene deletion within a single asexual erythrocytic growth cycle. Mol. Microbiol. 88, 687-701 (2013).

25. Banaszynski, L. A., Chen, L.-C., Maynard-Smith, L. A., Ooi, A. G. L. \& Wandless, T. J. A rapid, reversible, and tunable method to regulate protein function in living cells using synthetic small molecules. Cell 126, 995-1004 (2006).

26. Dvorin, J. D. et al. A plant-like Kinase in Plasmodium falciparum regulates parasite egress from erythrocytes. Science 328, 910-912 (2010).

27. Armstrong, C. M. \& Goldberg, D. E. An FKBP destabilization domain modulates protein levels in Plasmodium falciparum. Nat. Methods 4, 1007-1009 (2007).

28. Sassoon, I. et al. Regulation of Saccharomyces cerevisiae kinetochores by the type 1 phosphatase Glc7p. Genes Dev. 13, 545-555 (1999).

29. Yeoh, S. et al. Subcellular discharge of a serine protease mediates release of invasive malaria parasites from host erythrocytes. Cell 131, 1072-1083 (2007).

30. Thomas, J. A. et al. A protease cascade regulates release of the human malaria parasite Plasmodium falciparum from host red blood cells. Nat. Microbiol. 3, 447-455 (2018).

31. Collins, C. R. et al. Malaria Parasite cGMP-dependent protein kinase regulates blood stage merozoite secretory organelle discharge and egress. PLoS Pathog. 9, e1003344-13 (2013).

32. Glushakova, S. et al. Rounding precedes rupture and breakdown of vacuolar membranes minutes before malaria parasite egress from erythrocytes. Cell. Microbiol. 20, e12868 (2018).

33. Lehár, J., Stockwell, B. R., Giaever, G. \& Nislow, C. Combination chemical genetics. Nat. Chem. Biol. 4, 674-681 (2008).

34. Yokoyama, D. et al. Modulation of the growth of Plasmodium falciparum in vitro by protein serine/threonine phosphatase inhibitors. Biochem. Biophys. Res. Commun. 247, 18-23 (1998).

35. Treeck, M., Sanders, J. L., Elias, J. E. \& Boothroyd, J. C. The phosphoproteomes of Plasmodium falciparum and toxoplasma gondii reveal unusual adaptations within and beyond the parasites' boundaries. Cell Host Microbe 10, 410-419 (2011).

36. Pease, B. N. et al. Characterization of Plasmodium falciparum atypical kinase PfPK7- dependent phosphoproteome. J. Proteome Res. 17, 2112-2123 (2018).

37. Alam, M. M. et al. Phosphoproteomics reveals malaria parasite Protein Kinase $\mathrm{G}$ as a signalling hub regulating egress and invasion. Nat. Commun. 6, 7285 (2015).

38. Kumar, S. et al. PfCDPK1 mediated signaling in erythrocytic stages of Plasmodium falciparum. Nat. Commun. 8, 63 (2017).

39. Wang, W., Stukenberg, P. T. \& Brautigan, D. L. Phosphatase inhibitor-2 balances protein phosphatase 1 and aurora B kinase for chromosome segregation and cytokinesis in human retinal epithelial cells. Mol. Biol. Cell 19, 4852-4862 (2008).

40. Peggie, M. W. et al. Essential functions of Sds22p in chromosome stability and nuclear localization of PP1. J. Cell Sci. 115, 195-206 (2002).

41. Tung, H. Y., Wang, W. \& Chan, C. S. Regulation of chromosome segregation by Glc8p, a structural homolog of mammalian inhibitor 2 that functions as both an activator and an inhibitor of yeast protein phosphatase 1. Mol. Cell. Biol. 15, 6064-6074 (1995).

42. Salcedo-Sora, J. E., Caamano-Gutierrez, E., Ward, S. A. \& Biagini, G. A. The proliferating cell hypothesis: a metabolic framework for Plasmodium growth and development. Trends Parasitol. 30, 170-175 (2014).

43. Goto, H., Yasui, Y., Nigg, E. A. \& Inagaki, M. Aurora-B phosphorylates Histone $\mathrm{H} 3$ at serine 28 with regard to the mitotic chromosome condensation. Genes Cells 7, 11-17 (2002).

44. Mund, T., Lewis, M. J., Maslen, S. \& Pelham, H. R. Peptide and small molecule inhibitors of HECT-type ubiquitin ligases. Proc. Natl Acad. Sci. 111, 16736-16741 (2014)

45. Taylor, H. M. et al. The malaria parasite cyclic GMP-dependent protein kinase plays a central role in blood-stage schizogony. Eukaryot. Cell 9, 37-45 (2010).

46. Carucci, D. J. et al. Guanylyl cyclase activity associated with putative bifunctional integral membrane proteins in Plasmodium falciparum. J. Biol. Chem. 275, 22147-22156 (2000).

47. Bisio, H., Lunghi, M., Brochet, M. \& Soldati-Favre, D. Phosphatidic acid governs natural egress in Toxoplasma gondii via a guanylate cyclase receptor platform. Nat. Microbiol. 4, 420-428 (2019).

48. Brown, K. M. \& Sibley, L. D. Essential cGMP signaling in toxoplasma is initiated by a hybrid P-type ATPase-guanylate cyclase. Cell Host Microbe $\mathbf{2 4}$, 804-816 (2018).

49. Günay-Esiyok, Ö., Scheib, U., Noll, M. \& Gupta, N. An unusual and vital protein with guanylate cyclase and P4-ATPase domains in a pathogenic protist. Life Sci. Alliance 2, e201900402-e201900419 (2019).

50. Yang, L. et al. An apically located hybrid guanylate cyclase-ATPase is critical for the initiation of Ca $2+$ signaling and motility in Toxoplasma gondii. J. Biol. Chem. 294, 8959-8972 (2019).

51. Yuasa, K. et al. PfPDE1, a novel cGMP-specific phosphodiesterase from the human malaria parasite Plasmodium falciparum. Biochem. J. 392, 221-229 (2005).

52. Brochet, M. et al. Phosphoinositide metabolism links cGMP-dependent protein kinase $\mathrm{G}$ to essential $\mathrm{Ca} 2+$ signals at key decision points in the life cycle of malaria parasites. PLoS Biol. 12, e1001806-e1001815 (2014).

53. Baker, D. A. et al. Cyclic nucleotide signalling in malaria parasites. Open Biol. 7, 170213-170218 (2017).

54. Sah, R. K., Garg, S., Dangi, P., Ponnusamy, K. \& Singh, S. Phosphatidic acid homeostasis regulated by a type- 2 phosphatidic acid phosphatase represents a novel druggable target in malaria intervention. Cell Death Discov. 5, 107 (2019).

55. Bullen, H. E. et al. Phosphatidic acid-mediated signaling regulates microneme secretion in toxoplasma. Cell Host Microbe 19, 349-360 (2016).

56. Hidaka, H. et al. Specific, rapid, and sensitive enzymatic measurement of sphingomyelin, phosphatidylcholine and lysophosphatidylcholine in serum and lipid extracts. Clin. Biochem. 41, 1211-1217 (2008).

57. Hojjati, M. R. \& Jiang, X.-C. Rapid, specific, and sensitive measurements of plasma sphingomyelin and phosphatidylcholine. J. Lipid Res. 47, 673-676 (2006).

58. Jacot, D. et al. An apicomplexan actin-binding protein serves as a connector and lipid sensor to coordinate motility and invasion. Cell Host Microbe 20, 731-743 (2016).

59. Brancucci, N. M. B. et al. Lysophosphatidylcholine regulates sexual stage differentiation in the human malaria parasite Plasmodium falciparum. Cell 171, 1532-1544 (2017).

60. Wein, S. et al. Contribution of the precursors and interplay of the pathways in the phospholipid metabolism of the malaria parasite. J. Lipid Res. 59, 1461-1471 (2018)

61. Peter-Riesch, B., Fathi, M., Schlegel, W. \& Wollheim, C. B. Glucose and carbachol generate 1,2-diacylglycerols by different mechanisms in pancreatic islets. J. Clin. Invest. 81, 1154-1161 (1988).

62. Glushakova, S. et al. New stages in the program of malaria parasite egress imaged in normal and sickle erythrocytes. Curr. Biol. 20, 1117-1121 (2010).

63. Hale, V. L. et al. Parasitophorous vacuole poration precedes its rupture and rapid host erythrocyte cytoskeleton collapse in Plasmodium falciparum egress. Proc. Natl Acad. Sci. 114, 3439-3444 (2017).

64. Holm, P. I., Ueland, P. M., Kvalheim, G. \& Lien, E. A. Determination of choline, betaine, and dimethylglycine in plasma by a high-throughput method based on normal-phase chromatography-tandem mass spectrometry. Clin. Chem. 49, 286-294 (2003).

65. Zhang, M., Mishra, S., Sakthivel, R., Fontoura, B. M. A. \& Nussenzweig, V. UIS2: a unique phosphatase required for the development of Plasmodium liver stages. PLoS Pathog. 12, e1005370-20 (2016).

66. Absalon, $\mathrm{S}$. et al. Calcium-dependent protein kinase 5 is required for release of egress-specific organelles in Plasmodium falciparum. mBio 9, 411-416 (2018).

67. Raj, D. K. et al. Antibodies to PfSEA-1 block parasite egress from RBCs and protect against malaria infection. Science 344, 871-877 (2014).

68. Wiesner, S. et al. Autoinhibition of the HECT-Type Ubiquitin Ligase Smurf2 through Its C2 Domain. Cell 130, 651-662 (2007). 
69. Ichimura, T. et al. 14-3-3 proteins modulate the expression of epithelial $\mathrm{Na}$ +Channels by Phosphorylation-dependent Interaction with Nedd4-2 Ubiquitin Ligase. J. Biol. Chem. 280, 13187-13194 (2005).

70. Taylor, C. J., McRobert, L. \& Baker, D. A. Disruption of a Plasmodium falciparum cyclic nucleotide phosphodiesterase gene causes aberrant gametogenesis. Mol. Microbiol. 69, 110-118 (2008).

71. Moon, R. W. et al. A cyclic GMP signalling module that regulates gliding motility in a malaria parasite. PLoS Pathog. 5, e1000599-14 (2009).

72. Lakshmanan, V. et al. Cyclic GMP balance is critical for malaria parasite transmission from the mosquito to the mammalian host. mBio 6, e02330 (2015).

73. Roger, N. et al. Characterization of a 225 kilodalton rhoptry protein of Plasmodium falciparum. Mol. Biochemical Parasitol. 27, 135-141 (1988).

74. Yang, W. et al. Investigating Protein-Ligand interactions with a mutant FKBP possessing a designed specificity pocket. J. Med. Chem. 43, 1135-1142 (2000).

75. Nkrumah, L. J. et al. Efficient site-specific integration in Plasmodium falciparum chromosomes mediated by mycobacteriophage Bxb1 integrase. Nat. Methods 3, 615-621 (2006).

76. Przyborski, J. M. et al. Trafficking of STEVOR to the Maurer's clefts in Plasmodium falciparum-infected erythrocytes. The. EMBO J. 24, 2306-2317 (2005).

77. Trager, W. \& Jensen, J. B. Human malaria parasites in continuous culture. Science 193, 673-675 (1976)

78. Fidock, D. A. \& Wellems, T. E. Transformation with human dihydrofolate reductase renders malaria parasites insensitive to WR99210 but does not affect the intrinsic activity of proguanil. Proc. Natl Acad. Sci. 94, 10931-10936 (1997).

79. Triglia, T., Wang, P., Sims, P. F., Hyde, J. E. \& Cowman, A. F. Allelic exchange at the endogenous genomic locus in Plasmodium falciparum proves the role of dihydropteroate synthase in sulfadoxine-resistant malaria. EMBO J. 17, 3807-3815 (1998).

80. Paul, A. S. et al. Parasite calcineurin regulates host cell recognition and attachment by apicomplexans. Cell Host Microbe 18, 49-60 (2015).

81. Boyle, M. J. et al. Isolation of viable Plasmodium falciparum merozoites to define erythrocyte invasion events and advance vaccine and drug development. Proc. Natl Acad. Sci. 107, 14378-14383 (2010).

82. Schindelin, J. et al. Fiji: an open-source platform for biological-image analysis. Nat. Methods 9, 676-682 (2012).

83. Bei, A. K. et al. A flow cytometry-based assay for measuring invasion of red blood cells by Plasmodium falciparum. Am. J. Hematol. 85, 234-237 (2010).

84. Farrell, A. et al. A DOC2 protein identified by mutational profiling is essential for apicomplexan parasite exocytosis. Science 335, 218-221 (2012).

85. Ganter, M. et al. Plasmodium falciparum CRK4 directs continuous rounds of DNA replication during schizogony. Nat. Microbiol. 2, 17017 (2017).

86. Wessel, D. \& Flügge, U. I. A method for the quantitative recovery of protein in dilute solution in the presence of detergents and lipids. Anal. Biochem. 138, 141-143 (1984).

87. Paulo, J. A. \& Gygi, S. P. A comprehensive proteomic and phosphoproteomic analysis of yeast deletion mutants of 14-3-3 orthologs and associated effects of rapamycin. Proteomics 15, 474-486 (2015).

88. Paulo, J. A., Navarrete-Perea, J., Erickson, A. R., Knott, J. \& Gygi, S. P. An internal standard for assessing phosphopeptide recovery from metal ion/oxide enrichment strategies. J. Am. Soc. Mass Spectrom. 29, 1505-1511 (2018).

89. McAlister, G. C. et al. Increasing the multiplexing capacity of TMTs using reporter ion isotopologues with isobaric masses. Anal. Chem. 84, 7469-7478 (2012).

90. Ting, L., Rad, R., Gygi, S. P. \& Haas, W. MS3 eliminates ratio distortion in isobaric multiplexed quantitative proteomics. Nat. Methods 8, 937-940 (2011).

91. McAlister, G. C. et al. MultiNotch MS3 enables accurate, sensitive, and multiplexed detection of differential expression across cancer cell line proteomes. Anal. Chem. 86, 7150-7158 (2014).
92. Paulo, J. A., O’Connell, J. D. \& Gygi, S. P. A triple knockout (TKO) proteomics standard for diagnosing ion interference in isobaric labeling experiments. J. Am. Soc. Mass Spectrom. 27, 1620-1625 (2016).

93. Huttlin, E. L. et al. A tissue-specific atlas of mouse protein phosphorylation and expression. Cell 143, 1174-1189 (2010).

94. Vizcaíno, J. A. et al. 2016 update of the PRIDE database and its related tools Nucleic Acids Res. 44, D447-D456 (2016).

\section{Acknowledgements}

We thank the Harvard Electron Microscopy Facility and the Montpellier Ressources Imagerie [member of the national infrastructure France-BioImaging infrastructure supported by the French National Research Agency (ANR-10-INSB-04, Investments for the future)] for imaging. We thank the laboratories of Drs. Jeffrey Dvorin, Dyann Wirth, Barbara Burleigh, for use of equipment and reagents, as well as discussions. We thank Drs. Julian Rayner, Marcus Lee, Anthony Holder, and Michael Blackman for strains and reagents. This work was supported by NIH R21 AI128480 (M.T.D.), NIH R01 AI138551 (M.T.D.), NIH GM132129 (J.A.P.), NIH GM67945 (S.P.G), Laboratoire d'Excellence (LabEx) EpiGenMed ANR-10-LABX-12-01 (M.L., C.B.B.), Fondation pour la Recherche Médicale (Equipe FRM EQ20170336725, M.L.).

\section{Author contributions}

M.H.L. and M.T.D. jointly conceived and supervised the study; A.S.P., A.M., J.A.P., A.M B., L.B., M.S., A.L.K., B.E., J.S.N.A. and M.H.L., carried out experiments; A.S.P., A.M., J.M.G., M.S., C.B.B., M.L. and M.H.L. analyzed the data; A.S.P., J.A.P., S.P.G., M.H.L. and M.T.D., designed experiments; A.S.P., M.H.L. and M.T.D., wrote the manuscript.

\section{Competing interests}

The authors declare no competing interests.

\section{Additional information}

Supplementary information is available for this paper at https://doi.org/10.1038/s41467020-17306-1.

Correspondence and requests for materials should be addressed to M.H.L. or M.T.D.

Peer review information Nature Communications thanks David Baker and the other, anonymous, reviewer(s) for their contribution to the peer review of this work.

Reprints and permission information is available at http://www.nature.com/reprints

Publisher's note Springer Nature remains neutral with regard to jurisdictional claims in published maps and institutional affiliations.

(c) (i) Open Access This article is licensed under a Creative Commons Attribution 4.0 International License, which permits use, sharing, adaptation, distribution and reproduction in any medium or format, as long as you give appropriate credit to the original author(s) and the source, provide a link to the Creative Commons license, and indicate if changes were made. The images or other third party material in this article are included in the article's Creative Commons license, unless indicated otherwise in a credit line to the material. If material is not included in the article's Creative Commons license and your intended use is not permitted by statutory regulation or exceeds the permitted use, you will need to obtain permission directly from the copyright holder. To view a copy of this license, visit http://creativecommons.org/ licenses/by/4.0/.

(C) The Author(s) 2020 